\title{
22. PETROGRAPHICAL AND CHEMICAL STUDY OF BASEMENT BASALTS FROM THE GALAPAGOS SPREADING CENTER, LEG 701
}

\author{
Christine Laverne, Laboratoire de Géologie, BP 5, 97490 Ste. Clotilde (Réunion), France DOM \\ and \\ Gérard Vivier, Institut Dolomieu, L.A. CNRS nº 69, 38031 Grenoble Cedex, France
}

\section{INTRODUCTION}

During Leg 70, basalts from the region adjacent to the Galapagos Spreading Center were recovered at four sites (Sites 506, 507, 508, and 510). Previous to this, Leg 54 of the Deep Sea Drilling Project (DSDP) had recovered basalts from the same general area-at Sites 424 and 425 (Fig. 1).

The objectives of this chapter are the following:

1) To present the mineralogical and chemical features of fresh basalts recovered during Leg 70 in the Galapagos Rift area;

2) To compare these basalts with those recovered on Leg 54 and to complete a general model for the origin of basalts recovered from the Galapagos Spreading Center;

3) To assess changes in the mineralogical and chemical composition of these oceanic lavas brought about primarily by their reaction with seawater;

4) To determine whether a relationship exists among the ages of the basalts, their degree of fractionation, and their alteration.

\section{ANALYTICAL TECHNIQUES}

Minerals were identified by polarizing microscopy and analyzed by electron microprobe. Computerized electron-microprobe analyses were carried out at the Centre Océanologique de Bretagne, Brest (Automated Camebax). During the analyses, the accelerating voltage was kept at $15.0 \mathrm{kV}$. The electron-beam incidence of the Camebax has a take-off angle of about $42^{\circ}$, and the limit of detection is about 500 ppm. The precision of the method used is about $1 \%$.

Major, minor, and trace elements were analyzed in bulk-rock samples (after the usual grinding and homogenization in an agate mortar) by X-ray fluorescence (CGR Alpha 2020; rhodium tube), at the Institut Dolomieu, Grenoble.

\section{FRESH ROCK PETROGRAPHY}

Basalt textures and estimated amounts of magmatic minerals are summarized in Table 1 . Young crust at Sites 506,507 , and 508 yielded nearly identical samples of fineto medium-grained, aphyric to sparsely plagioclase (or, more rarely, pyroxene) phyric basalts. Textures range from glassy to variolitic to subophitic, with the grain size of the groundmass minerals increasing with distance from the glassy rims, as is usually observed in oceanic basalts. Plagioclase $\left(\mathrm{An}_{63}\right.$; see analysis 1 , Table 2$)$ is the dominant phenocryst phase $(<5 \%)$, with clinopyroxene phenocrysts less abundant $(<4 \%)$. Plagioclase and

\footnotetext{
${ }^{1}$ Honnorez, J., Von Herzen, R. P., et al., Init. Repts. DSDP, 70: Washington (U.S. Govt. Printing Office)
}

clinopyroxene appear also as microlites and in rocks with quenched textures. Olivine was not observed in these basalts. Titanomagnetite is the dominant opaque phase $(10-15 \%)$ of the bulk rock, and primary spherules of sulfide minerals occur. Vesicles range from $1 \%$ to $8 \%$ by volume.

Site 510 is characterized by fine- to medium-grained, moderately (3-10\%) plagioclase phyric, sparsely $(1 \%)$ olivine phyric basalt. The olivine is $\mathrm{Mg}$ rich $\left(\mathrm{FO}_{86-87}\right.$; see analyses 2, 3, 4, Table 2). Titanomagnetite is less abundant $(5-8 \%)$ than in basalts from the other sites, and no vesicles have been observed.

Comparison of the phenocryst assemblages and $\mathrm{Fe}-$ $\mathrm{Ti}$ oxide abundances seems to indicate that basalts from Site 510 are more primitive than are those from Sites 506,507 , and 508 . This is confirmed by our study of bulk-rock chemistry.

\section{BULK COMPOSITION}

The samples which we analyzed chemically were, as often as possible, pairs of the same hand specimen which exhibited (1) apparently fresh rock and (2) altered rim. Homogeneous samples of "fresh" rock were easily isolated.

Whole-rock analyses and normative mineralogy of four samples from Site 510 and eight samples from Sites 506,507 , and 508 are presented in Table 3. For comparison with "parental and differentiated magmas," all the analyses have been normalized to $100 \%$ after water was removed and all iron reduced (see Table 4).

Site 510. Fresh basalt samples from Site 510 have high $\mathrm{Al}_{2} \mathrm{O}_{3}$ (15.07 to 16.35 wt.\%), $\mathrm{MgO}$ (7.81-8.49 wt. \%), $\mathrm{CaO}(12.91-13.21 \mathrm{wt} . \%)$ and relatively high $\mathrm{Ni}$ (157-170 ppm), Cr (406-446 ppm), and $\mathrm{Cu}$ (100-110 ppm) contents.

The high $\mathrm{MgO}$ content and the presence of normative olivine $\left(\mathrm{Fo}_{66}\right)$ in one sample corresponded to the petrographic observation of modal olivine $\left(\mathrm{Fo}_{78-80}\right)$. Olivine contains 200 to $400 \mathrm{ppm}$ of $\mathrm{Ni}$ and up to $900 \mathrm{ppm}$ of $\mathrm{Cr}$ (see Table 3). Normative quartz ranges between $0.00 \%$ and $0.75 \%$.

The chemical composition of Site 510 basalts is very close to that of the parental magma of the Galapagos Spreading Center described by Clague and Bunch (1976), with $\mathrm{FeO} * / \mathrm{MgO}=0.99$ to 1.06 and $\mathrm{MgO}$ content $=$ $7.81 \%$ to $8.49 \%$.

High $\mathrm{Cr}$ and $\mathrm{Ti}$ content and low $\mathrm{Sr}$ ( 53 to $57 \mathrm{ppm}$ ), $\mathrm{Ba}$ (13 to $19 \mathrm{ppm}$ ), and V (264 to $275 \mathrm{ppm}$ ) content reflect a 


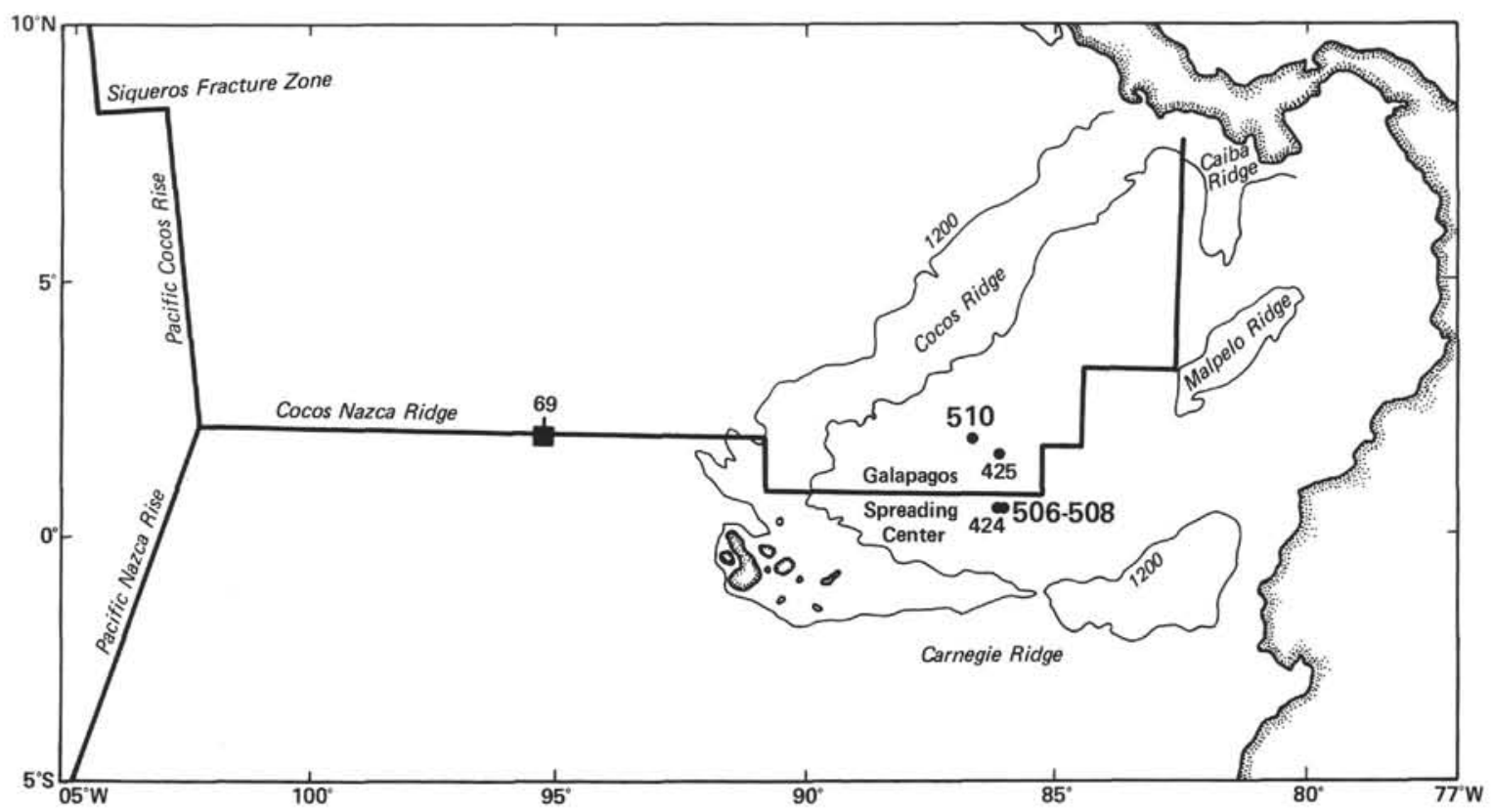

Figure 1. Location of Sites 506, 507, 508, and 510 in the region adjacent to the Galapagos Spreading Center. Also shown are Sites 424 and 425 , Leg 54 .

Table 1. Textures, magmatic minerals (estimated percentages), and secondary-mineral occurrence in basalts from Sites 506, 507, 508, and 510 .

\begin{tabular}{|c|c|c|c|c|c|c|c|c|c|c|c|c|c|c|c|c|c|}
\hline \multirow{3}{*}{$\begin{array}{c}\text { Sample } \\
\text { (interval in } \mathrm{cm} \text { ) }\end{array}$} & \multirow[b]{3}{*}{ Texture } & \multicolumn{3}{|c|}{ Phenocrysts } & \multicolumn{5}{|c|}{ Groundmass } & \multicolumn{8}{|c|}{ Secondary Minerals } \\
\hline & & \multirow[t]{2}{*}{ ol } & \multirow{2}{*}{$\begin{array}{l}\operatorname{cpx} \\
(\%)\end{array}$} & \multirow[t]{2}{*}{ pl } & \multirow[t]{2}{*}{ ol } & \multirow[t]{2}{*}{$\operatorname{cpx}$} & \multirow{2}{*}{$\begin{array}{l}\text { pl } \\
(\%)\end{array}$} & \multirow{2}{*}{$\operatorname{tm}$} & \multirow[t]{2}{*}{ ve/vo } & \multicolumn{8}{|c|}{ Clay Minerals ${ }^{\mathrm{a}}$} \\
\hline & & & & & & & & & & 1 & 2 & 3 & 4 & 5 & 6 & 7 & c \\
\hline $506 \mathrm{G}-2-1,10-14$ & Hyalopilitic & - & - & - & - & 45 & 40 & 15 & & & & & & & & & \\
\hline $506 \mathrm{G}-2-1,49-52$ & $\begin{array}{l}\text { Subvariolitic } \\
\text { Glomerophyric }\end{array}$ & & & & & & & & & & & & & & & & \\
\hline & $\begin{array}{l}\text { Glomerophyric } \\
\text { Hyalopilitic }\end{array}$ & - & 2 & 2 & - & 40 & 40 & 12 & 2 (ve) & $\mathrm{x}$ & & & & & & & \\
\hline $506 \mathrm{G}-2-1,126-123$ & $\begin{array}{l}\text { Hyalopilitic } \\
\text { Glomerophyric }\end{array}$ & - & - & 1 & - & 43 & 43 & 12 & 1 (ve) & & & & & & & & \\
\hline $507 \mathrm{~B}-1-1,29-32$ & Subophitic to inter- & & & & & & & & & & & & & & & & \\
\hline $\begin{array}{l}507 \mathrm{C}-3-1,2-5 \\
508 \mathrm{~B}-3-1,12-15\end{array}$ & Hyalopilitic & - & - & - & - & 40 & 40 & 13 & 7 (ve) & $x$ & $\mathrm{x}$ & & & & & & \\
\hline $\begin{array}{l}508 \mathrm{~B}-3-1,12-15 \\
508 \mathrm{~B}-3-1,32-34\end{array}$ & $\begin{array}{l}\text { Hyalopilitic } \\
\text { Hyalopilitic }\end{array}$ & - & 4 & 4 & $\bar{z}$ & $\begin{array}{l}37 \\
40\end{array}$ & $\begin{array}{l}37 \\
40\end{array}$ & $\begin{array}{l}10 \\
14\end{array}$ & 8 (ve) & $x$ & $\mathrm{x}$ & & & & & & \\
\hline $508 \mathrm{~B}-3-1,43-46$ & Hyalopilitic & - & - & - & - & 40 & $\begin{array}{l}40 \\
40\end{array}$ & $\begin{array}{l}14 \\
14\end{array}$ & $\begin{array}{l}6 \text { (ve) } \\
6 \text { (ve) }\end{array}$ & $\begin{array}{l}x \\
x\end{array}$ & $\mathrm{x}$ & $\mathrm{x}$ & & & & & \\
\hline $510-9-1,4-7$ & Hyalopilitic & & & & & & & & & & & & & & & & \\
\hline & Subglomerophyric & - & - & 7 & 5 & 35 & 45 & 7 & 1 (vo) & & & & $\mathrm{x}$ & $\mathrm{x}$ & & & \\
\hline $510-9-1,44-46$ & $\begin{array}{l}\text { Hyalopilitic } \\
\text { Subolomerophyric }\end{array}$ & & & & & & & & & & & & & & & & \\
\hline $510-9-3,31-36$ & $\begin{array}{l}\text { Subgiomerophyric } \\
\text { Hyalopilitic }\end{array}$ & 1 & - & 10 & 7 & 31 & 41 & 8 & 2 (vo) & & & & $\mathrm{x}$ & $x$ & $\mathrm{x}$ & & \\
\hline & Subglomerophyric & - & - & 6 & 5 & 40 & 42 & 5 & 2 (vo) & $\mathrm{x}$ & & & & $\mathrm{x}$ & $\mathrm{x}$ & $\mathrm{x}$ & $\mathrm{x}$ \\
\hline $510-11-1,79-82$ & Hyalopilitic & & & & & & & & & & & & & & & & \\
\hline & Subglomerophyric & 1 & - & 3 & 3 & 43 & 44 & 5 & 1 (vo) & $\mathrm{x}$ & & & & $\mathrm{x}$ & & & \\
\hline
\end{tabular}

Note: $\mathrm{tm}=$ titanomagnetite; $\mathrm{ve} / \mathrm{vo}=$ vesicles and voids; $\mathrm{pl}=$ plagioclase; $\mathrm{cpx}=$ clinopyroxene; $\mathrm{ol}=$ olivine; and $\mathrm{c}=$ carbonate. a Clay minerals are numbered according to Table 3 .

very low degree of (or even no) fractionation from the parental magma.

Site 507 and 508. Basalts from Sites 507 and 508 are richer in $\mathrm{Na}, \mathrm{K}, \mathrm{Ti}, \mathrm{P}, \mathrm{Mn}, \mathrm{V}$, and $\mathrm{Sr}$ than are those from Site 510. Their higher $\mathrm{SiO}_{2}$ content and normative quartz amount $(1.92-4.17 \%)$ identify them as quartz tholeiites.

They are very rich in $\mathrm{FeO}^{*}$ and $\mathrm{TiO}_{2}$ (11.81-13.11 wt. \% and 1.82-2.09 wt.\%, respectively) and correlatively rich in $\mathrm{V}$ (379-409 ppm) and poor in $\mathrm{Al}_{2} \mathrm{O}_{3}$ $(12.69-13.40 \%)$. They can, therefore, be classified as ferrobasalts (Melson et al., 1976) and are similar to other ferrobasalts already dredged from the area of the Galapagos Spreading Center (Anderson et al., 1975; Schilling et al., 1976; Clague and Bunch, 1976; Fodor et al., 1980).
The medium content of $\mathrm{Ni}(118-148 \mathrm{ppm})$ and $\mathrm{Co}(61-$ $103 \mathrm{ppm})$ and the high $\mathrm{FeO} * / \mathrm{MgO}(1.77$ to 1.97$)$ ratio indicate a substantial fractionation of Fo-rich olivine from the parental magma. The simultaneous destabilization of $\mathrm{Cr}$ spinets with this olivine would explain the relatively low $\mathrm{Cr}$ (110-164 ppm) content observed.

Compared with Site 510 basalts, the fractionation corresponds also to higher contents of $\mathrm{Sr}$ (75-87 ppm), $\mathrm{Ba}$ (23-48 ppm), and $\mathrm{Na}_{2} \mathrm{O}$ (2.29-244 wt. \% instead of $1.84-1.88$ wt. \%) and lower $\mathrm{CaO}(10.53-10.79$ wt. \% instead of 12.91-13.21 wt.\%).

Site 506. Basalts from Site 506 are also ferrobasalts and are very similar to those from Sites 507 and 508 . However they are slightly poorer in $\mathrm{TiO}_{2}$ (1.56-1.78 wt. \%), $\mathrm{MgO}$ (7.17-7.28 wt.\%), V (347-375 ppm), and $\mathrm{Cr}(176-190 \mathrm{ppm})$ than the latter. Normative quartz is 
Table 2. Microprobe analyses of magmatic minerals.

\begin{tabular}{|c|c|c|c|c|}
\hline \multirow[b]{2}{*}{ Component } & \multicolumn{4}{|c|}{$\begin{array}{c}\text { Sample } \\
\text { (interval in } \mathrm{cm} \text { ) }\end{array}$} \\
\hline & $\begin{array}{c}507 \mathrm{C}-3-1 \\
2-5 \\
\text { (Plagioclase) }\end{array}$ & $\begin{array}{c}\text { 510-9-1, } \\
\text { 94-96 } \\
\text { (Olivine) }^{\mathrm{a}}\end{array}$ & $\begin{array}{c}\text { 510-9-1, } \\
\text { 94-96 } \\
\text { (Olivine) }^{\mathrm{a}}\end{array}$ & $\begin{array}{c}\text { 510-9-3, } \\
31-36 \\
\text { (Olivine) }\end{array}$ \\
\hline \multicolumn{5}{|c|}{ Major oxides (wt. \%) } \\
\hline $\mathrm{SiO}_{2}$ & 53.74 & 40.58 & 40.68 & 40.91 \\
\hline $\mathrm{Al}_{2} \mathrm{O}_{3}$ & 29.15 & 0.02 & 0.04 & 0.03 \\
\hline $\mathrm{FeO}^{*}$ & 0.78 & 12.63 & 12.80 & 12.98 \\
\hline $\mathrm{MnO}$ & - & 0.15 & 0.11 & 0.18 \\
\hline $\mathrm{MgO}$ & 0.29 & 47.14 & 47.03 & 46.64 \\
\hline $\mathrm{CaO}$ & 12.42 & 0.34 & 0.29 & 0.31 \\
\hline $\mathrm{Na}_{2} \mathrm{O}$ & 4.00 & - & 0.01 & 0.04 \\
\hline $\mathrm{K}_{2} \mathrm{O}$ & - & - & - & 0.04 \\
\hline $\mathrm{TiO}_{2}$ & 0.02 & - & - & - \\
\hline $\mathrm{NiO}$ & - & 0.13 & 0.13 & - \\
\hline $\mathrm{Cr}_{2} \mathrm{O}_{3}$ & 0.03 & 0.03 & 0.05 & - \\
\hline $\mathrm{P}_{2} \mathrm{O}_{5}$ & 0.23 & 0.03 & - & - \\
\hline Total & 100.65 & 101.06 & 101.14 & 101.12 \\
\hline \multicolumn{5}{|c|}{ Cation proportions } \\
\hline $\mathrm{Si}$ & 2.418 & 0.997 & 0.995 & 1.005 \\
\hline $\mathrm{Al}$ & 1.546 & 0.001 & 0.001 & 0.001 \\
\hline $\mathrm{Fe}$ & 0.029 & 0.260 & 0.263 & 0.267 \\
\hline Mn & - & 0.003 & 0.002 & 0.004 \\
\hline $\mathrm{Mg}$ & 0.019 & 1.727 & 1.722 & 1.708 \\
\hline $\mathrm{Ca}$ & 0.598 & 0.009 & 0.008 & 0.008 \\
\hline $\mathrm{Na}$ & 0.349 & - & - & 0.002 \\
\hline $\mathrm{Ti}$ & - & - & - & - \\
\hline $\mathrm{Ni}$ & - & 0.002 & 0.003 & - \\
\hline $\mathrm{Cr}$ & 0.001 & - & 0.001 & - \\
\hline $\mathrm{P}$ & 0.009 & 0.001 & - & - \\
\hline Total & 4.969 & 3.001 & 2.999 & 2.996 \\
\hline An & 63.19 & & & \\
\hline $\mathrm{Ab}$ & 36.81 & & & \\
\hline Or & - & & & \\
\hline Fo & - & 86.68 & 86.54 & 86.33 \\
\hline $\mathrm{Fa}$ & - & 13.32 & 13.46 & 13.67 \\
\hline
\end{tabular}

also definitely less abundant (1.54-1.91\%). These features allow us to classify Site 506 basalts as intermediate between those from Site 510 and those from Sites 507 and 508. This intermediate character appears in all the diagrams illustrating the relationship among the fresh basalts or their alteration states (Fig. 2, 6, 7).

\section{Comparison with Site $\mathbf{4 2 4}$ and $\mathbf{4 2 5}$}

Fodor et al. (1980) described basalts from Sites 424 and 425 , which were drilled during Leg 54 in the area of the Galapagos Spreading Center. Site 424 is located near Sites 506, 507, and 508, whereas Site 425 is located near Site 510 (Fig. 1).

A comparison with average normalized analyses of parental and differentiated basalts from the Galapagos Spreading Center proposed by Clague and Bunch (1976) and with basalts from Sites 424 and 425 is presented in Table 4.

Site 510 basalts are very close to the parental magma. Site $\mathbf{4 2 5}$ basalts represent moderately fractionated oceanic tholeiites by mainly plagioclase plus olivine fractionation in the proportion 3:1 (crystallized: $28 \%$, Fodor et al., 1980).
Basalts from Sites 507, 508, and 424 are close to differentiate 3 (Clague and Bunch, 1976). Up to $60 \%$ of the parental magma has fractionally crystallized to form these ferrobasalts. For Clague and Bunch (1976) these ferrobasalts are formed by fractionation of plagioclase, clinopyroxene, and olivine in the proportions 9.3:7.7:1 (differentiate 3 ). The calculated proportions given by Fodor et al. (1980) are respectively $11.3: 12.8: 1$ and $50 \%$ of crystallized magma for the same ferrobasalts.

From Figure 2 and Table 4, one may infer that the Site 425 parental magma corresponds to a tholeiite whose composition is very close to those of basalts described above. Fractionation would result in ferrobasalt (Sites 424, 507, 508), and Site 506 basalts would be an intermediate, close to those from Sites 507 and 508 . Thus, fractionation increases from Site 510 to Site 424 basalts as follows: $510,425,506,507-508,424$.

A linear correlation exists between the paleomagnetic age and the degree of fractionation of basalts from the Galapagos Spreading Center except for those from Site 506. The exceptional feature of Site 506 basalts will be further underlined in the alteration study. As shown in Figure 2, the more evolved basalts are the youngest.

\begin{tabular}{lcccccc}
\hline Site & 510 & 425 & 506 & 507 & 508 & 424 \\
\hline Age (m.y.) & 2.73 & 1.80 & 0.54 & 0.69 & 0.85 & 0.62 \\
\hline
\end{tabular}

\section{COLORED ALTERATION HALOS}

In basalts from the Galapagos Spreading Center, alteration appears as colored halos which are subparallel to exposed surfaces and fissures. The halos are darker than the fresh basalt and thus were called "black halos" on the ship.

Most basalt samples from Site 506 are fresh, but a thin $(<4 \mathrm{~mm})$ alteration halo occurs in a few samples. At Site 507, the texture of the basalt clearly controls the alteration. Subophitic basalts are completely fresh whereas those that are hyalopilitic show dark alteration halos (maximum thickness: $15 \mathrm{~mm}$ ). At Sites 508 and 510, all of the samples exhibit thick altered halos ranging, respectively, up to $50 \mathrm{~mm}$ and from 5 to $40 \mathrm{~mm}$.

Thus, the widths of the altered halos increase with age from Site 506 to $508-$ i.e., between 0.55 and 0.85 m.y. (Fig. 3).

\section{SECONDARY MINERALS}

Alteration halos in basalts from Sites 506, 507, 508, and 510 are characterized (both in hand specimens and under the microscope) by secondary minerals in vesicles and interstitial voids. In addition, olivine is replaced by secondary minerals in Site 510 basalts. Feldspar and pyroxene are always fresh.

Because of the small size and number of samples, it was not always possible to make a thin section representative of a complete alteration rim. Nevertheless, we identified various kinds of clay minerals, whose characteristics are summarized in Table 5 . We have chosen to represent their chemistry on a $\mathrm{FeO}^{*}-\mathrm{MgO}-\mathrm{K}_{2} \mathrm{O}$ triangle (Fig. 4), for the behavior of these elements during alteration is particularly interesting. Because of the lack 
Table 3. Bulk-sample chemical analyses (major elements in wt.\%, monocations in \%, and CIPW norms).

\begin{tabular}{|c|c|c|c|c|c|c|c|c|c|c|c|c|c|c|c|c|c|c|c|c|c|}
\hline \multirow[b]{2}{*}{ Component } & \multicolumn{21}{|c|}{$\begin{array}{c}\text { Sample } \\
\text { (interval in } \mathrm{cm} \text { ) }\end{array}$} \\
\hline & $\begin{array}{c}506 \mathrm{G}-2-1, \\
10-14^{\mathrm{a}}\end{array}$ & $\begin{array}{l}506 \mathrm{G}-2-1-1 \\
49-52 \mathrm{~b}\end{array}$ & $\begin{array}{c}506 \mathrm{G}-2-1-1, \\
49-52^{\mathrm{a}}\end{array}$ & $\begin{array}{l}506 \mathrm{G}-2-1 \cdot 1, \\
121-123^{\mathrm{a}}\end{array}$ & $\begin{array}{c}507 \mathrm{~B}-1-1,1, \\
29-32^{\mathrm{a}}\end{array}$ & $\begin{array}{c}507 C-3-1, \\
2-5 b\end{array}$ & $\begin{array}{c}507 \mathrm{C}-3-1, \\
2-5^{\mathrm{a}}\end{array}$ & $\begin{array}{c}508 \mathrm{~B}-3-1,1 \\
12-15 \mathrm{~b}\end{array}$ & $\begin{array}{c}508 \mathrm{~B}-3-1, \\
12-15^{\mathrm{a}}\end{array}$ & $\begin{array}{c}508 \mathrm{~B}-3-1-1 \\
32-34 \mathrm{~b}^{\circ}\end{array}$ & $\begin{array}{c}508 \mathrm{~B}-3-1-1, \\
32-34^{\mathrm{a}}\end{array}$ & $\begin{array}{c}508 \mathrm{~B}-3-1, \\
43-46^{\mathrm{b}}\end{array}$ & $\begin{array}{c}508 \mathrm{~B}-3-1, \\
43-46^{\mathrm{a}}\end{array}$ & $\underset{4 \rightarrow-7 b}{510-9-1,}$ & $\underset{47^{\mathrm{a}}}{510-9.1}$ & $\begin{array}{l}510-9-1, \\
44-46^{6}\end{array}$ & $\begin{array}{l}510-9-1,1, \\
44-46^{a}\end{array}$ & $\begin{array}{l}510-9-3, \\
31-36^{b}\end{array}$ & $\begin{array}{l}510-9-3, \\
31-36^{\mathrm{a}}\end{array}$ & $\begin{array}{c}510-11-1, \\
79-82^{b}\end{array}$ & $\begin{array}{c}510-11-1, \\
79-82^{\mathrm{a}}\end{array}$ \\
\hline \multicolumn{22}{|c|}{ Major oxide (wt.\%) } \\
\hline $\mathrm{SiO}_{2}$ & 49.83 & 49.66 & 50.29 & 50.71 & 48.93 & 48.80 & 50.64 & 48.31 & 50.42 & 49.05 & 51.07 & 49.63 & 49.71 & 49.08 & 49.89 & 49.23 & 49.92 & 49.04 & 49.36 & 49.18 & 49.63 \\
\hline $\mathrm{Al}_{2} \mathrm{O}_{3}$ & 13.56 & 13.60 & 13.55 & 13.80 & 12.69 & 12.98 & 13.35 & 12.58 & 13.05 & 13.01 & 13.39 & 12.87 & 13.40 & 15.22 & 15.84 & 15.52 & 16.35 & 15.53 & 16.08 & 15.16 & 15.07 \\
\hline $\mathrm{Fe}_{2} \mathrm{O}_{3}$ & 2.06 & 2.22 & 1.85 & 1.86 & 2.73 & 5.75 & 2.40 & 5.05 & 2.57 & $\begin{array}{l}6.03 \\
875\end{array}$ & 2.72 & 4.72 & 2.20 & 2.54 & 1.48 & 2.72 & 1.70 & 2.96 & 1.75 & 2.83 & 1.53 \\
\hline $\begin{array}{ll}\mathrm{FeO} \\
\mathrm{MnO}\end{array}$ & $\begin{array}{l}9.90 \\
0.22\end{array}$ & 9.91 & $\begin{array}{r}10.10 \\
0.18\end{array}$ & 9.81 & $\begin{array}{l}10.66 \\
0.22\end{array}$ & 8.35 & 10.11 & 9.00 & 10.44 & $\begin{array}{l}8.75 \\
024\end{array}$ & 9.44 & 9.12 & 9.83 & 6.65 & 7.04 & 6.41 & 6.78 & 6.24 & 6.76 & 6.41 & 7.00 \\
\hline $\begin{array}{l}\mathrm{MnO} \\
\mathrm{MBO}\end{array}$ & $\begin{array}{l}0.22 \\
7.23\end{array}$ & $\begin{array}{l}0.21 \\
7.17\end{array}$ & $\begin{array}{l}0.18 \\
7.12\end{array}$ & $\begin{array}{l}0.19 \\
7.28\end{array}$ & $\begin{array}{l}0.22 \\
6.64\end{array}$ & $\begin{array}{l}0.17 \\
5.68\end{array}$ & $\begin{array}{l}0.23 \\
6.46\end{array}$ & $\begin{array}{l}0.21 \\
6.39\end{array}$ & $\begin{array}{l}0.28 \\
6.57\end{array}$ & $\begin{array}{l}0.24 \\
6.27\end{array}$ & $\begin{array}{l}0.26 \\
6.63\end{array}$ & $\begin{array}{l}0.21 \\
6.51\end{array}$ & $\begin{array}{l}0.22 \\
6.66\end{array}$ & $\begin{array}{l}0.18 \\
7.59\end{array}$ & $\begin{array}{l}0.15 \\
8.01\end{array}$ & $\begin{array}{l}0.15 \\
7.55\end{array}$ & $\begin{array}{l}0.18 \\
7.81\end{array}$ & $\begin{array}{l}0.14 \\
7.92\end{array}$ & $\begin{array}{l}0.14 \\
8.13\end{array}$ & $\begin{array}{l}0.16 \\
8.30\end{array}$ & $\begin{array}{l}0.16 \\
8.49\end{array}$ \\
\hline $\mathrm{CaO}$ & 11.10 & 11.14 & 11.16 & 11.24 & 10.53 & 9.85 & 10.64 & 10.09 & 10.53 & 9.97 & 10.79 & 10.30 & 10.79 & 12.78 & 13.09 & 12.78 & 13.21 & 12.98 & 13.12 & 12.80 & 12.91 \\
\hline $\mathrm{Na}_{2} \mathrm{O}$ & 2.26 & 2.28 & 2.24 & 2.27 & 2.36 & 2.45 & 2.44 & 2.29 & 2.30 & 2.30 & 2.40 & 2.18 & 2.29 & 1.79 & 1.84 & 1.80 & 1.86 & 1.85 & 1.88 & 1.84 & 1.86 \\
\hline $\mathrm{K}_{2} \mathrm{O}$ & 0.13 & 0.10 & 0.15 & 0.13 & 0.14 & 0.32 & 0.07 & 0.35 & 0.17 & 0.50 & 0.20 & 0.49 & 0.24 & 0.20 & 0.04 & 0.25 & 0.05 & 0.18 & 0.05 & 0.26 & 0.05 \\
\hline $\mathrm{TiO}_{2}$ & 1.78 & 1.65 & 1.56 & 1.58 & 1.90 & 1.85 & 1.89 & 1.96 & 2.09 & 2.05 & 2.08 & 1.71 & 1.82 & 0.80 & 0.79 & 0.77 & 0.83 & 0.79 & 0.81 & 0.81 & 0.85 \\
\hline $\begin{array}{l}\mathrm{P}_{2} \mathrm{O}_{5} \\
\mathrm{H}_{2} \mathrm{O}\end{array}$ & $\begin{array}{l}0.07 \\
1.66\end{array}$ & $\begin{array}{l}0.08 \\
1.15\end{array}$ & $\begin{array}{l}0.11 \\
1.46\end{array}$ & $\begin{array}{l}0.09 \\
1.10\end{array}$ & $\begin{array}{l}0.13 \\
1.68\end{array}$ & $\begin{array}{l}0.12 \\
3.29\end{array}$ & $\begin{array}{l}0.14 \\
0.86\end{array}$ & $\begin{array}{l}0.15 \\
2.53\end{array}$ & $\begin{array}{l}0.16 \\
1.61\end{array}$ & $\begin{array}{l}0.17 \\
1.87\end{array}$ & $\begin{array}{l}0.17 \\
0.98\end{array}$ & $\begin{array}{l}0.14 \\
2.17\end{array}$ & $\begin{array}{l}0.13 \\
1.52\end{array}$ & $\begin{array}{l}0.03 \\
2.30\end{array}$ & $\begin{array}{l}\text { tr } \\
1.17\end{array}$ & $\begin{array}{l}0.02 \\
1.31\end{array}$ & $\begin{array}{l}\text { tr } \\
1.07\end{array}$ & $\begin{array}{l}0.02 \\
1.57\end{array}$ & $\begin{array}{l}0.02 \\
1.94\end{array}$ & $\begin{array}{l}\text { tr } \\
0.93\end{array}$ & $\begin{array}{l}\text { tr } \\
1.24\end{array}$ \\
\hline Total & 99.80 & 99.17 & 99.77 & 100.06 & 98.61 & 99.61 & 99.23 & 98.91 & 100.19 & 100.21 & 100.13 & 100.05 & 98.81 & 99.16 & 99.34 & 98.51 & 99.76 & 99.22 & 100.04 & 98.68 & 98.79 \\
\hline \multicolumn{22}{|c|}{ Trace Elements (ppm) } \\
\hline $\mathrm{Ba}$ & 28 & 26 & 30 & 27 & 23 & 28 & 31 & 24 & 30 & 24 & 41 & 29 & 28 & 16 & 18 & 15 & 15 & 14 & 13 & 14 & 19 \\
\hline Co & 114 & 193 & 105 & 97 & 69 & 141 & 61 & 120 & 103 & 93 & 98 & 126 & 90 & 100 & 68 & 125 & 81 & 79 & 64 & 76 & 84 \\
\hline $\mathrm{Cr}$ & 190 & 193 & 184 & 176 & 117 & 110 & 110 & 155 & 156 & 146 & 164 & 129 & 126 & 418 & 416 & 399 & 422 & 411 & 406 & 410 & 446 \\
\hline $\mathrm{Cu}$ & 73 & 77 & 80 & 82 & 54 & 54 & 56 & 53 & 52 & 53 & 57 & 59 & 66 & 101 & 110 & 94 & 100 & 94 & 104 & 82 & 107 \\
\hline $\mathrm{Ni}$ & 151 & 148 & 141 & 140 & 117 & 178 & 122 & 116 & 148 & 128 & 148 & 130 & 133 & 155 & 157 & 150 & 169 & 151 & 157 & 150 & 170 \\
\hline $\mathrm{Sr}_{\mathrm{y}}$ & $\begin{array}{r}83 \\
375\end{array}$ & $\begin{array}{r}72 \\
355\end{array}$ & $\begin{array}{r}73 \\
347\end{array}$ & $\begin{array}{r}75 \\
349\end{array}$ & $\begin{array}{r}73 \\
381\end{array}$ & ${ }_{371}^{76}$ & $\begin{array}{r}75 \\
381\end{array}$ & $\begin{array}{r}68 \\
376\end{array}$ & 76 & 73 & $\begin{array}{r}87 \\
409\end{array}$ & $\begin{array}{r}71 \\
363\end{array}$ & $\begin{array}{r}80 \\
379\end{array}$ & $\begin{array}{r}58 \\
267\end{array}$ & $\begin{array}{l}57 \\
266\end{array}$ & $\begin{array}{r}54 \\
264\end{array}$ & 55 & 53 & 53 & 54 & 53 \\
\hline $\begin{array}{l}\mathrm{V} \\
\mathrm{Rb}\end{array}$ & $\begin{array}{r}375 \\
<10\end{array}$ & $\begin{array}{l}355 \\
<10\end{array}$ & $\begin{array}{l}347 \\
<10\end{array}$ & $\begin{array}{r}349 \\
<10\end{array}$ & $\begin{array}{r}381 \\
<10\end{array}$ & ${ }_{11}^{371}$ & $\begin{array}{l}381 \\
<10\end{array}$ & $\begin{array}{l}376 \\
<10\end{array}$ & $\begin{array}{l}403 \\
<10\end{array}$ & $\begin{array}{l}394 \\
13\end{array}$ & $\begin{array}{r}409 \\
<10\end{array}$ & $\begin{array}{l}363 \\
20\end{array}$ & $\begin{array}{r}379 \\
<10\end{array}$ & $\begin{array}{l}267 \\
<10\end{array}$ & $\begin{array}{l}266 \\
<10\end{array}$ & $\begin{array}{l}264 \\
<10\end{array}$ & $\begin{array}{l}275 \\
<10\end{array}$ & $\begin{array}{l}2733 \\
<10\end{array}$ & $\begin{array}{r}273 \\
<10\end{array}$ & $\begin{array}{l}269 \\
<10\end{array}$ & $\begin{array}{r}268 \\
<10\end{array}$ \\
\hline $\mathrm{FeO}^{*}$ & 11.75 & 11.91 & 11.77 & $\begin{array}{l}<10 \\
11.48\end{array}$ & ${ }_{13.12}^{<10}$ & $\begin{array}{l}11 \\
13.53\end{array}$ & $\begin{array}{l}<10 \\
12.27\end{array}$ & $\begin{array}{l}<10 \\
13.55\end{array}$ & $\begin{array}{l}<10 \\
12.75\end{array}$ & $\begin{array}{l}13 \\
14.18\end{array}$ & $\begin{array}{l}<10 \\
11.88\end{array}$ & $\begin{array}{l}20 \\
13.37\end{array}$ & $\begin{array}{l}<10 \\
11.81\end{array}$ & $\begin{array}{l}<.94 \\
8.94\end{array}$ & $<100$ & $<8.86$ & $<8.31$ & $<10$ & $<8.34$ & $<100$ & $\begin{array}{c}<10 \\
8.38\end{array}$ \\
\hline $\mathrm{FeO} * / \mathrm{MgO}$ & 1.63 & 1.66 & 1.65 & 1.58 & 1.98 & 2.38 & 1.90 & 2.12 & 1.94 & 2.26 & 1.79 & 2.05 & 1.77 & 1.18 & $\begin{array}{l}8.31 \\
1.04\end{array}$ & $\begin{array}{l}0.00 \\
1.17\end{array}$ & $\begin{array}{l}8.06 \\
1.06\end{array}$ & $\begin{array}{l}0.120 \\
1.12\end{array}$ & 1.03 & $\begin{array}{l}8.00 \\
1.08\end{array}$ & $\begin{array}{l}0.99 \\
0.99\end{array}$ \\
\hline$O_{\dot{x}}^{\circ}$ & 0.160 & 0.168 & 0.142 & 0.146 & 0.187 & 0.383 & 0.176 & 0.336 & 0.181 & 0.383 & 0.206 & 0.318 & 0.168 & 0.256 & 0.159 & 0.276 & 0.184 & 0.299 & 0.189 & 0.284 & 0.164 \\
\hline \multicolumn{22}{|l|}{ Cattion (\%) } \\
\hline & 47.71 & 47.61 & 48.07 & 48.08 & 47.73 & 48.21 & 48.59 & 47.61 & 48.41 & 47.50 & 48.60 & 48.11 & 48.13 & 47.19 & 47.13 & 47.15 & 46.93 & 46.68 & 46.61 & 46.72 & 47.12 \\
\hline $\mathrm{Al}_{3+1}$ & 15.30 & 15.37 & 15.26 & $\begin{array}{l}15.42 \\
15.42\end{array}$ & 14.59 & $\begin{array}{l}15.11 \\
15.42\end{array}$ & $\begin{array}{l}15.10 \\
70.10\end{array}$ & 14.61 & $\begin{array}{l}14.77 \\
14.71\end{array}$ & 14.85 & $\begin{array}{l}75.02 \\
15.02\end{array}$ & $\begin{array}{l}14.71 \\
\end{array}$ & 15.29 & 17.25 & 17.63 & 17.52 & 18.11 & 17.42 & 17.89 & 16.97 & 16.86 \\
\hline $\mathrm{Fe}_{2+}^{3+}$ & 1.48 & 1.60 & 1.33 & 1.33 & 2.00 & 4.27 & 1.73 & 3.75 & 1.86 & 4.39 & 1.95 & 3.44 & 1.60 & 1.84 & 1.05 & 1.96 & 1.20 & 2.12 & 1.24 & 2.02 & 1.09 \\
\hline $\mathrm{Fe}^{2+}$ & 7.93 & 7.95 & 8.07 & 7.78 & 8.70 & 6.90 & 8.11 & 7.42 & 8.38 & 7.09 & 7.51 & 7.39 & 7.96 & 5.35 & 5.56 & 5.13 & 5.33 & 4.97 & 5.34 & 5.09 & 5.56 \\
\hline $\mathrm{Mn}$ & $\begin{array}{r}0.18 \\
0.32\end{array}$ & 0.17 & 0.15 & $\begin{array}{r}0.15 \\
1029\end{array}$ & $\begin{array}{l}0.18 \\
9.65\end{array}$ & $\begin{array}{l}0.14 \\
8.36\end{array}$ & $\begin{array}{l}0.19 \\
9.24\end{array}$ & $\begin{array}{l}0.18 \\
9.39\end{array}$ & $\begin{array}{l}0.23 \\
9.40\end{array}$ & 0.20 & 0.21 & 0.17 & 0.18 & 0.15 & 0.12 & 0.12 & $\begin{array}{r}0.14 \\
10.94\end{array}$ & 0.11 & 0.11 & 0.13 & 0.13 \\
\hline $\begin{array}{l}\mathrm{Mg} \\
\mathrm{Ca}\end{array}$ & $\begin{array}{l}10.32 \\
11.39\end{array}$ & $\begin{array}{l}{ }_{10.25}^{11.44} \\
\end{array}$ & $\begin{array}{l}{ }_{10.14}^{10.43} \\
11.45\end{array}$ & $\begin{array}{l}10.29 \\
11.42\end{array}$ & $\begin{array}{r}9.65 \\
11.01\end{array}$ & $\begin{array}{r}8.36 \\
\end{array}$ & $\begin{array}{r}9.24 \\
10.94\end{array}$ & $\begin{array}{r}9.39 \\
10.65\end{array}$ & $\begin{array}{r}9.40 \\
10.83\end{array}$ & $\begin{array}{r}9.05 \\
10.35\end{array}$ & $\begin{array}{r}9.41 \\
1100\end{array}$ & $\begin{array}{r}9.41 \\
10.70\end{array}$ & $\begin{array}{r}9.61 \\
11.19\end{array}$ & $\begin{array}{l}10.88 \\
13.17\end{array}$ & $\begin{array}{l}11.28 \\
13.25\end{array}$ & $\begin{array}{l}10.78 \\
13.11\end{array}$ & $\begin{array}{l}10.94 \\
13.30\end{array}$ & $\begin{array}{l}11.24 \\
13.24\end{array}$ & $\begin{array}{l}11.44 \\
13.27\end{array}$ & $\begin{array}{l}\begin{array}{l}11.75 \\
13.03\end{array} \\
13\end{array}$ & $\begin{array}{l}{ }_{13}^{12.01} \\
13.13\end{array}$ \\
\hline $\begin{array}{l}\mathrm{Ca} \\
\mathrm{Na}\end{array}$ & 4.20 & 4.24 & $\begin{array}{r}11.43 \\
4.15\end{array}$ & 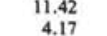 & $\begin{array}{r}11.01 \\
4.46\end{array}$ & $\begin{array}{r}10.43 \\
4.69\end{array}$ & $\begin{array}{r}10.94 \\
4.54\end{array}$ & $\begin{array}{r}10.65 \\
4.38\end{array}$ & $\begin{array}{r}10.83 \\
4.28\end{array}$ & $\begin{array}{r}10.35 \\
4.32\end{array}$ & $\begin{array}{r}11.00 \\
4.43\end{array}$ & $\begin{array}{r}10.70 \\
4.10\end{array}$ & $\begin{array}{r}11.19 \\
4.30\end{array}$ & $\begin{array}{r}13.17 \\
3.34\end{array}$ & $\begin{aligned} 13.25 \\
3.37\end{aligned}$ & $\begin{array}{l}13.11 \\
3.34\end{array}$ & $\begin{array}{r}13.30 \\
3.39\end{array}$ & $\begin{array}{r}13.24 \\
3.41\end{array}$ & $\begin{array}{l}13.27 \\
3.44\end{array}$ & $\begin{array}{l}1.03 \\
3.39\end{array}$ & $\begin{array}{r}13.13 \\
3.42\end{array}$ \\
\hline $\mathrm{K}$ & 0.16 & 0.12 & 0.18 & 0.16 & 0.17 & 0.40 & $\begin{array}{l}4.54 \\
0.09\end{array}$ & $\begin{array}{l}4.38 \\
0.44\end{array}$ & 0.21 & $\begin{array}{l}4.32 \\
0.62\end{array}$ & $\begin{array}{l}4.43 \\
0.24\end{array}$ & $\begin{array}{l}4.61 \\
0.61\end{array}$ & 0.30 & 0.25 & 0.05 & 0.31 & 0.06 & $\begin{array}{l}. .41 \\
0.22\end{array}$ & 0.06 & 0.32 & 0.06 \\
\hline $\mathrm{Ti}$ & 1.28 & 1.19 & 1.12 & 1.13 & 1.39 & 1.37 & 1.36 & 1.45 & 1.51 & 1.49 & 1.49 & 1.25 & 1.33 & 0.58 & 0.56 & 0.55 & 0.59 & 0.57 & 0.58 & 0.58 & 0.61 \\
\hline$\stackrel{P}{\Sigma}=100^{\mathrm{P}}$ & 0.06 & 0.06 & 0.09 & 0.07 & 0.11 & 0.10 & 0.11 & 0.13 & 0.13 & 0.14 & 0.14 & 0.11 & 0.11 & 0.02 & tr & 0.02 & tr & 0.02 & 0.02 & tr & tr \\
\hline \multicolumn{22}{|l|}{ CIPW Norm } \\
\hline q & 1.54 & 1.37 & 1.82 & 1.91 & 1.92 & 5.83 & 3.56 & 4.22 & 3.76 & 5.14 & 4.17 & 4.63 & 2.43 & 1.67 & 0.68 & 1.75 & 0.75 & 1.03 & - & 0.59 & 0.31 \\
\hline or & 0.76 & 0.59 & 0.88 & 0.76 & 0.82 & 1.89 & 0.41 & 2.06 & 1.00 & 2.95 & & & & & & 1.47 & 0.29 & 1.06 & 0.29 & 1.53 & 0.29 \\
\hline$a b$ & 19.12 & 19.29 & 19.95 & 19.20 & 19.96 & 20.73 & 20.64 & 19.37 & 19.46 & 19.46 & 20.30 & 18.44 & 19. & 15.14 & 15. & 15.23 & 15.73 & 15.65 & 15.90 & 15.56 & 15.73 \\
\hline an & 26.47 & 26.57 & 26.47 & 27.08 & 23.61 & 23.47 & 25.26 & 23.01 & 24.78 & 23.69 & 25.17 & 23. & 25. & 32.9 & 34. & 33.52 & 36.1 & 33.5 & 25.28 & 32.33 & 32.62 \\
\hline di & 23.08 & 23.11 & 23.16 & 23.05 & 22.88 & 19.98 & 21.90 & 21.21 & 21.74 & 19.98 & 22.31 & 21.45 & 22.28 & 24.51 & 24. & 24.01 & 23.83 & 24.73 & 24.00 & 25.09 & 25.43 \\
\hline hy & 20.61 & 20.52 & 21.10 & 21.02 & 19.84 & 12.28 & 19.18 & 15.09 & 19.76 & 14.07 & 17.70 & 16.15 & 19.26 & 16.17 & 18.78 & 15.72 & 17,90 & 15.78 & 18.37 & 16.98 & 19.31 \\
\hline ii & 3.38 & 3.13 & 2.96 & 3.00 & 3.60 & 3.51 & 3.58 & 3.72 & 3.96 & 3.89 & 2.95 & 3.24 & 3.45 & 1.51 & 1.50 & 1.46 & 1.57 & 1.50 & 1.53 & 1.53 & 1.61 \\
\hline $\mathrm{mt}$ & 2.98 & 3.21 & 2.68 & 2.69 & 3.95 & 8.33 & 3.47 & 7.32 & 3. & 8.74 & 3.94 & 6.84 & 3.18 & 3.68 & 2.14 & 3.94 & 2.46 & 4.29 & 2.53 & 4.10 & 2.21 \\
\hline ap & 0.16 & 0.18 & -0.26 & -0.21 & 0.30 & $\begin{array}{l}0.28 \\
-\end{array}$ & -0.33 & 0.35 & ${ }_{-}^{0.37}$ & -0.40 & 0.40 & 0.33 & 0.30 & 0.07 & $\overline{-}$ & 0.04 & $\overline{-}$ & ${ }_{-}^{0.04}$ & $\begin{array}{l}0.04 \\
0.10\end{array}$ & $\overline{-}$ & $\bar{z}$ \\
\hline
\end{tabular}

batesh. 
Table 4. Average normalized analyses of basalts from the Galapagos Spreading Center.

\begin{tabular}{|c|c|c|c|c|c|c|c|c|c|}
\hline $\begin{array}{c}\text { Oxide } \\
\text { (wt.\%) }\end{array}$ & Parent $^{\mathrm{a}}$ & Site 510 & Differentiate $1^{\mathrm{a}}$ & Site $425^{b}$ & Differentiate $2^{\mathrm{a}}$ & Site 506 & Site $507-508$ & Site $424^{b}$ & Differentiate $3^{\mathrm{a}}$ \\
\hline $\mathrm{SiO}_{2}$ & 50.37 & 50.73 & 50.27 & 52.08 & 50.19 & 51.18 & 51.25 & 51.45 & 51.30 \\
\hline $\mathrm{Al}_{2} \mathrm{O}_{3}$ & 15.99 & 16.16 & 15.52 & 14.42 & 14.43 & 13.88 & 13.46 & 13.46 & 13.19 \\
\hline $\mathrm{FeO}$ & 8.97 & 8.52 & 10.10 & 10.45 & 12.01 & 11.87 & 12.72 & 13.53 & 13.79 \\
\hline $\mathrm{MnO}$ & 0.15 & 0.16 & 0.16 & 0.17 & 0.18 & 0.20 & 0.25 & 0.21 & 0.20 \\
\hline $\mathrm{MgO}$ & 8.00 & 8.28 & 6.65 & 7.61 & 7.11 & 7.34 & 6.74 & 6.39 & 5.80 \\
\hline $\mathrm{CaO}$ & 12.84 & 13.35 & 12.28 & 11.94 & 11.19 & 11.36 & 10.88 & 10.41 & 10.60 \\
\hline $\mathrm{Na}_{2} \mathrm{O}$ & 2.45 & 1.90 & 3.13 & 2.13 & 2.70 & 2.30 & 2.43 & 2.48 & 2.60 \\
\hline $\mathrm{K}_{2} \mathrm{O}$ & 0.07 & 0.05 & 0.23 & 0.04 & 0.19 & 0.14 & 0.16 & 0.08 & 0.14 \\
\hline $\mathrm{TiO}_{2}$ & 1.09 & 0.84 & 1.58 & 1.05 & 1.86 & 1.67 & 1.99 & 1.88 & 2.17 \\
\hline $\mathrm{P}_{2} \mathrm{O}_{5}$ & 0.07 & tr. & 0.11 & 0.08 & 0.15 & 0.09 & 0.14 & 0.15 & 0.20 \\
\hline $\mathrm{FeO} / \mathrm{MgO}$ & 1.12 & 1.03 & 1.52 & 1.37 & 1.69 & 1.62 & 1.94 & 2.12 & 2.38 \\
\hline
\end{tabular}

of X-ray diffraction data we cannot precisely identify these clay minerals. But by comparing their chemistry and optical characteristics to other data (Bolhke et al., 1981; Honnorez et al., 1978; Mevel, 1980) we are able to assume that most of these clay minerals belong to the smectite group.

Site 506. In Sample 506G-2-1, 49-52 cm (analyzed for bulk rock chemistry), the only secondary mineral is a heterogeneous orange-to-brown material occurring in interstitial voids and in a very tiny vein. No vesicles were observed in the alteration halo. A vesicle in-filling with a center of orange material and a rim of greenish clay had been observed on board ship in Sample 506G-2-1, 90-93 cm.

Site 507. In Sample 507C-3-1, 2-5 cm, the vesicles and other voids are filled by yellowish green clay, by orange-to-brown material, or both; if by both, the orange brown mineral occurs in the vesicle rim, whereas the yellowish green one occurs in the center of the vesicle. The analyses of both materials occurring in the same vesicle are given in Table 6 (analyses 1 and 2). The yellowish green mineral is an $\mathrm{K}$, Fe-rich clay, chemically close to a nontronite.

The shipboard scientific party had first identified the orange to brown material as iron oxide and/or hydroxide. But it is too rich in silica (14-20\%) and poor in iron $\left(\mathrm{FeO}^{*}=50-63 \%\right)$ to be a pure hydroxide. On the other hand, it is too poor in silica and rich in iron to be a pure smectite. Thus, this material is probably a mixture of iron oxyhydroxides and clay mineral. The $\mathrm{K}, \mathrm{Fe}$-rich clay seems to have been formed before the orange brown material.

Site 508. The altered rim of Sample 508B-3-1, 12-15 $\mathrm{cm}$ clearly shows a zonational pattern. From the exterior to the center of the sample, one observes in the interstitial voids and vesicles: (1) brown to brownish green clay (analysis 5, Table 6), associated with an orange brown material. The clay is very $\mathrm{FeO}^{*}$ rich $(39 \%)$ and slightly $\mathrm{K}_{2} \mathrm{O}$ rich (3\%); (2) green to yellowish green clay (analysis 4, Table 6) in the vesicle rims, and orange material (analysis 3, Table 6) in the vesicle cores (Plate 1); (3) green to yellowish green clay (Plate 1); (4) nothing.

Based on both chemical analyses and the respective locations of these various minerals, we believe that the brownish green clay is a former yellowish green clay which has undergone an initial oxidation accompanied by loss of $\mathrm{SiO}_{2}$ and gain of $\mathrm{FeO}^{*}$. The arrow in Figure 4 indicates this trend.

The following succession of vesicle in-fillings was observed, from the exposed surfaces to the core of Sample 508B-3-1, 43-46 cm: (1) brownish black material; (2) brown material (Plate 2); (3) brownish orange material in the vesicle rims and brown material in center; (4) brownish green clay; (5) nothing.

Thus, if we consider the brownish green clay as a reference for the start of clay oxidation, one could say that secondary minerals of Sample 508B-3-1, 43-46 cm have all undergone oxidation, whereas in Sample 508B-3-1, $12-15 \mathrm{~cm}$, oxidation did not reach the innermost secondary products. Further, the brownish black material is perhaps chemically very close to an iron oxyhydroxide, suggesting that oxidation is more important in the more external part of the altered halo. Oxidation appears to have progressed toward the center of the basalt.

Site 510. Basalts from Site 510 contain olivine microphenocrysts and/or phenocrysts. Alteration appears not only as interstitial void in-fillings but also as a replacement of olivine.

In Sample 510-9-1, 94-96 cm, two zones are recognized in the alteration halo:

1) The outer zone contains olivine crystals whose cores are replaced by a $\mathrm{K}, \mathrm{Fe}$-poor, $\mathrm{Mg}$-rich pale green clay (analysis 10, Table 6), whereas their external part is composed of a K, Fe-rich, Mg-poor clay mineral (analysis 9, Table 6), chemically close to the "protoceladonite" found in Holes 417A, 417D, and 418A (Mevel, 1979). This replacement is sometimes very incomplete, with alteration products occurring only along cracks and around the olivine crystals. Olivine is sometimes completely replaced by clay minerals. Miarolitic voids are filled with the $\mathrm{Fe}$, K-rich dark green clay mineral (analysis 11, Table 6). These observations suggest that the Mgrich pale green clay mineral at Site 510 was able to form only because it could take $\mathrm{Mg}$ from the $\mathrm{Mg}$-rich olivine and also explain why the pale green clay mineral does not occur in basalts from Sites 506, 507, and 508 .

2) In the inner zone, olivine is replaced by a very pale brown clay mineral (analysis 12, Table 6), which is $\mathrm{MgO}$ rich $(17 \%), \mathrm{K}_{2} \mathrm{O}$ poor $(0.1 \%)$, and contains significant $\mathrm{Al}_{2} \mathrm{O}_{3}(2.6 \%)$. It also fills interstitial voids. Unfortunately, this mineral is unstable under the microprobe beam. Hence, its analysis must be regarded as semi- 

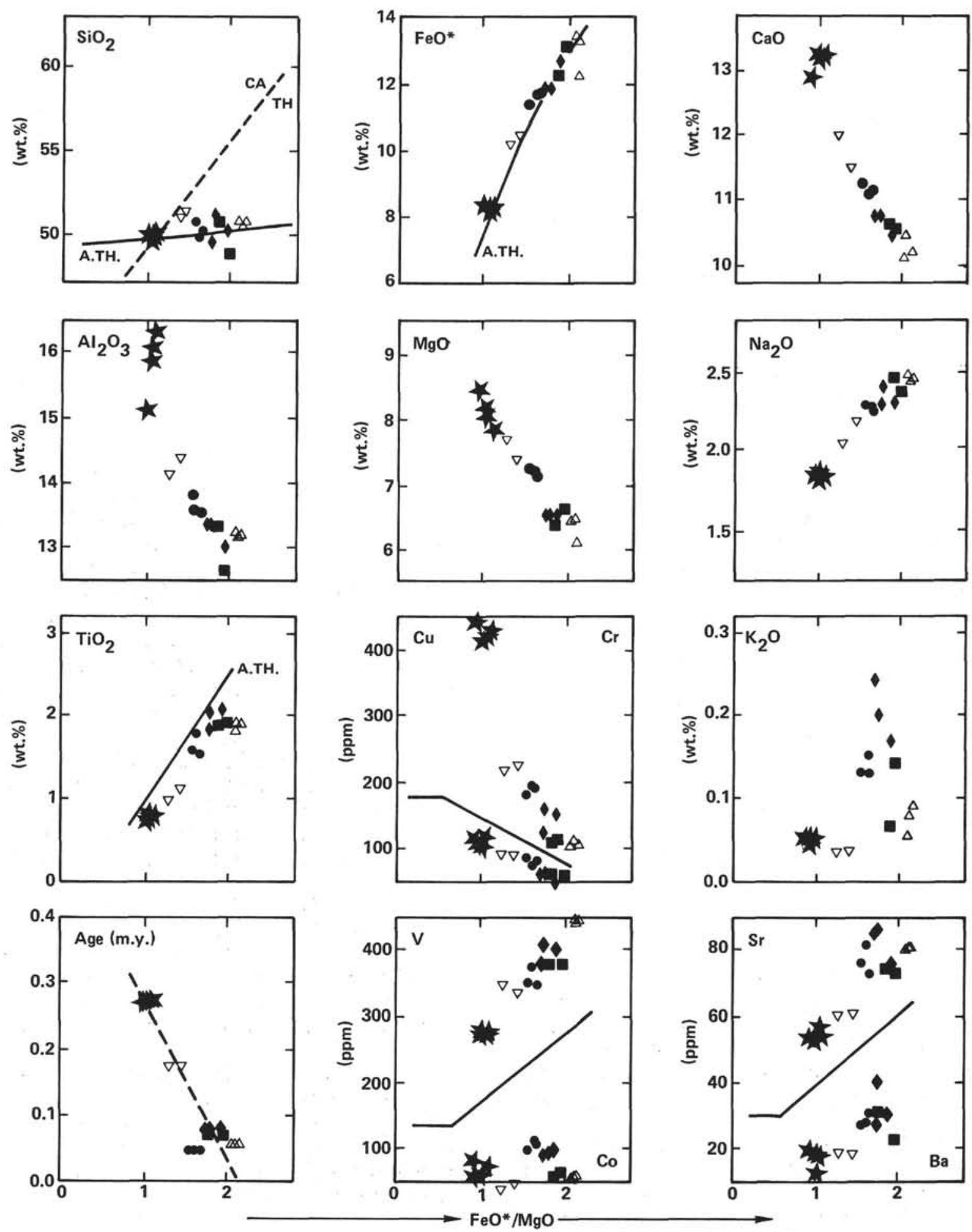

Legend $\bullet=$ Site $506 \square=$ Site $507 \downarrow=$ Site $508 \downarrow=$ Site $510 \Delta=$ Site $424 \nabla=$ Site 425

Figure 2. Bulk chemical variations in fresh rocks from the Galapagos Spreading Center: weight percent oxides vs. FeO*/MgO.

quantitative. A similar mineral, called "light tan smectite," is described by Bohkle et al. (1981) and Honnorez et al. (1978), in basalts from Leg 46, Hole 496B.

In Sample 510-9-3, 31-36 cm, we observed the most complete zonal alteration pattern. From the exposed surface to the core of the sample, the following sequence is found:

1) Orange to brown material;

2) Dark green clay mineral (analysis 13, Table 6) + orange brown material (Plate 2 ) \pm dark brown clay 


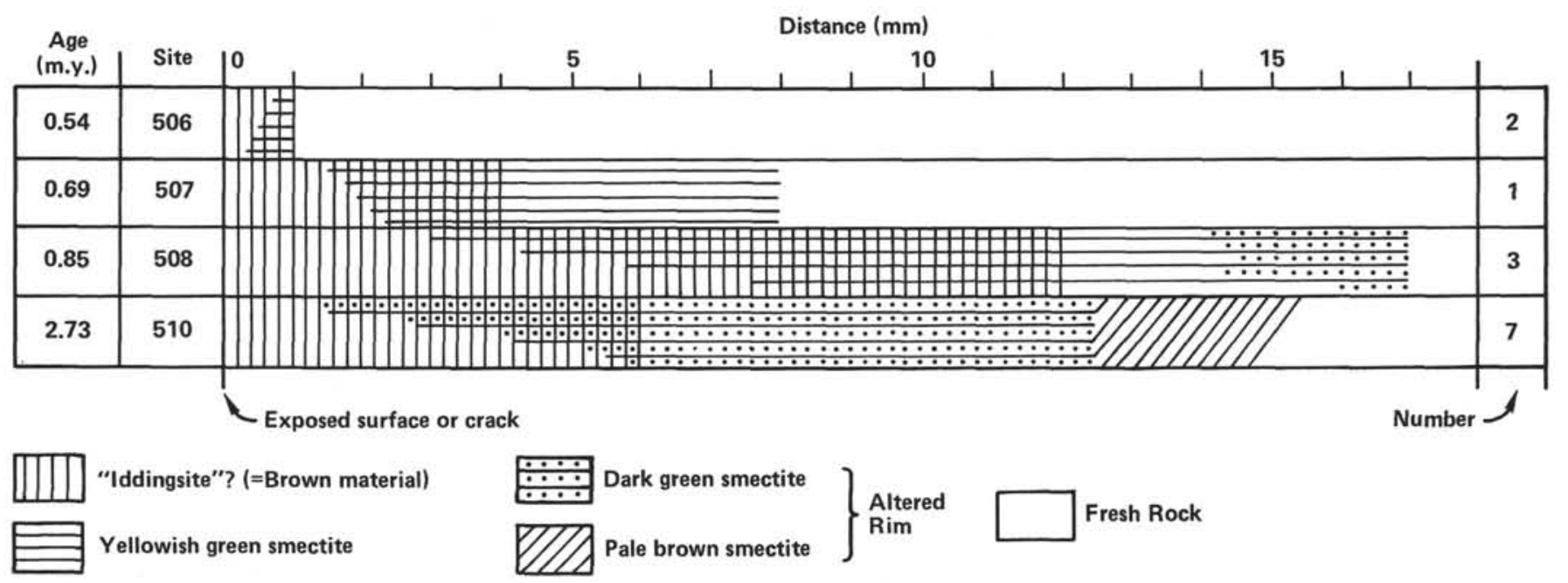

Figure 3. Variation in average thickness and nature of fillings in altered rim with age of rock.

Table 5. Features of clay minerals occurring in Leg 70 Galapagos basalts.

\begin{tabular}{|c|c|c|c|c|c|c|}
\hline Type & Color & $\begin{array}{c}\text { Color with } \\
\text { Crossed Polars }\end{array}$ & Habitus & $\begin{array}{l}\text { Mode of } \\
\text { Occurrence }\end{array}$ & $\begin{array}{r}\text { Principa } \\
\text { Chemical Fe: }\end{array}$ & tures \\
\hline 1 & Orange to brown & $\begin{array}{l}\text { Abnormal } \\
\text { dark orange or } \\
\text { dark brown }\end{array}$ & Granulous & ves-vo-ol & $\begin{array}{l}\text { Very high } \mathrm{FeO} \\
\mathrm{No}_{2} \mathrm{O} \\
\text { Low } \mathrm{SiO}_{2}\end{array}$ & $50-63 \%$ \\
\hline 2 & Yellowish green & $\begin{array}{l}\text { Abnormal } \\
\text { greenish gray }\end{array}$ & $\begin{array}{l}\text { Radial or not } \\
\text { radial needles }\end{array}$ & ves-vo & $\begin{array}{l}\text { Medium } \mathrm{FeO}^{*} \\
\text { Medium } \mathrm{K}_{2} \mathrm{O} \\
\mathrm{High} \mathrm{SiO}_{2}\end{array}$ & $\begin{array}{l}27-30 \% \\
2-3 \% \\
50 \%\end{array}$ \\
\hline 3 & Brownish green & $\begin{array}{l}\text { Abnormal dark } \\
\text { brownish gray }\end{array}$ & Small slabs & ves-vo & $\begin{array}{l}\mathrm{High} \mathrm{FeO}^{*} \\
\text { Medium } \mathrm{K}_{2} \mathrm{O} \\
\text { Medium } \mathrm{SiO}_{2}\end{array}$ & $\begin{array}{l}39 \% \\
3 \% \% \\
45 \%\end{array}$ \\
\hline 4 & Pale green & $\begin{array}{l}\text { Light gray to } \\
\text { yellow } \\
\text { (1st order) }\end{array}$ & Fibers & ol & $\begin{array}{l}\text { Low } \mathrm{FeO}^{*} \\
\text { Very low } \mathrm{K}_{2} \mathrm{O} \\
\mathrm{High} \mathrm{SiO}_{2}\end{array}$ & $\begin{array}{l}8-10 \% \\
1 \% \\
48 \%\end{array}$ \\
\hline 5 & Olive green & $\begin{array}{l}\text { Abnormal dark } \\
\text { olive to black }\end{array}$ & $\begin{array}{l}\text { Very tiny crystals } \\
\text { (granulous aspect) }\end{array}$ & ves-vo-ol & 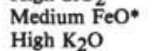 & $\begin{array}{l}26-28 \% \\
5-8 \%\end{array}$ \\
\hline 6 & Pale brown & $\begin{array}{l}\text { Abnormal } \\
\text { brownish gray }\end{array}$ & $\begin{array}{l}\text { Tiny slabs (slightly } \\
\text { granulous aspect) }\end{array}$ & vo-ol & $\begin{array}{l}\text { Low } \mathrm{FeO}^{*} \\
\text { No } \mathrm{K}_{2} \mathrm{O} \\
\text { Medium } \mathrm{SiO}_{2}\end{array}$ & $6 \%$ \\
\hline 7 & Dark brown & $\begin{array}{l}\text { Dark gray } \\
\text { to black }\end{array}$ & Slight granulous & ol & 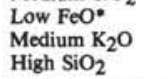 & $\begin{array}{l}24 \% \\
2-3 \% \\
52 \%\end{array}$ \\
\hline
\end{tabular}

Note: ves $=$ vesicle filling, vo $=$ interstitial void filling, ol $=$ olivine replacement.

mineral (analysis 16, Table 6) \pm carbonates (analysis 17, Table 6). The calcium carbonate is rich in both $\mathrm{MgO}$ $(2.10-2.50 \%)$ and $\mathrm{MnO}(1.22-1.74 \%)$;

3) Dark green clay minerals;

4) A very pale brown clay mineral (analysis 14 , Table $6) \pm$ calcium carbonate \pm pale greenish brown clay mineral (analysis 15, Table 6; see also Plate 3).

The pale brown clay mineral was not observed in other samples from Hole 510. Thus, freshly cored rock is directly in contact with the dark green clay mineral zone, which is without any doubt the more abundant alteration product in basalts from Hole 510 .

Figure 5 represents schematically the $\mathrm{FeO}^{*}, \mathrm{MgO}$, and $\mathrm{K}_{2} \mathrm{O}$ variations in the main clay minerals from Site 510 as a function of their distance to an exposed surface or crack.

The sequence from dark green clay mineral to brown material can be explained as following. During a first stage, a $\mathrm{K}, \mathrm{Fe}$-rich $/ \mathrm{Mg}$, Al-poor clay mineral would precipitate from percolating fluids in interstitial voids. The early formed clay minerals would be transformed later into the brown $\mathrm{Fe}$-rich material as a result of oxidation.
It is not possible to say whether the pale brown clay formed prior to or contemporaneously with the dark green clay.

\section{Comparison of Alteration at Site 506-508 and Site 510}

Three main features allow us to distinguish young basalts (Sites 506, 507, and 508) from older ones (Site 510) on the basis of their alteration (see Table 1):

1) Potassium-rich dark olive clay mineral is very rare in young basalts; however such basalts do contain a moderately rich-K, yellowish green clay. The first characteristic could be explained if the time of seawater-basalt interaction were too short to allow for the formation of a K-rich clay mineral.

2) The K-poor pale brown clay mineral was not observed at Sites 506, 507, and 508. The same explanation as above is proposed. On the other hand, the presence of this mineral could be related to bulk-rock chemistry-i.e., basalts from Site 510 are richer in $\mathbf{M g}$ than are those from Sites 506, 507, and 508.

3) Carbonates are frequent at Site 510, very rare at Site 508, and were not observed at Sites 506 and 507. 
Table 6. Selected microprobe analyses of secondary minerals.

\begin{tabular}{|c|c|c|c|c|c|c|c|c|c|c|c|c|c|c|c|c|c|}
\hline $\begin{array}{c}\text { Oxide } \\
\text { (wt.\%) }\end{array}$ & 1 & 2 & 3 & 4 & 5 & 6 & 7 & 8 & 9 & 10 & 11 & 12 & 13 & 14 & 15 & 16 & 17 \\
\hline $\mathrm{SiO}_{2}$ & 14.96 & 49.91 & 16.38 & 49.77 & 45.15 & 51.23 & 51.91 & 53.06 & 52.52 & 48.25 & 52.03 & 40.03 & 51.74 & 47.11 & 47.68 & 52.28 & 0.06 \\
\hline $\mathrm{Al}_{2} \mathrm{O}_{3}$ & 0.63 & 3.74 & 1.33 & 1.48 & 1.34 & 0.58 & 0.40 & 1.51 & 0.92 & 0.77 & 0.38 & 2.58 & 0.31 & 2.40 & 2.11 & 0.39 & - \\
\hline $\mathrm{FeO}^{*}$ & 49.25 & 30.69 & 63.51 & 27.78 & 39.08 & 28.23 & 8.62 & 26.71 & 26.46 & 9.90 & 29.38 & 5.82 & 25.79 & 8.69 & 12.55 & 24.24 & 0.50 \\
\hline $\mathrm{MnO}$ & 0.06 & 0.05 & 0.20 & 0.02 & 0.08 & 0.06 & - & 0.13 & - & 0.17 & 0.06 & 0.05 & 0.12 & 0.15 & 0.04 & 0.03 & 1.33 \\
\hline $\mathrm{MgO}$ & 1.79 & 2.87 & 1.33 & 1.55 & 1.81 & 5.46 & 19.73 & 5.59 & 5.45 & 16.92 & 4.06 & 16.69 & 4.62 & 19.73 & 18.39 & 10.36 & 2.33 \\
\hline $\mathrm{CaO}$ & 0.28 & 0.75 & 0.52 & 0.19 & 0.54 & 0.63 & 0.48 & 0.51 & 0.43 & 0.52 & 0.40 & 0.87 & 0.27 & 0.47 & 0.73 & 0.73 & 53.21 \\
\hline $\mathrm{Na}_{2} \mathrm{O}$ & 0.31 & 0.19 & 0.13 & 0.12 & 0.18 & 0.15 & 0.24 & 0.08 & 0.23 & 0.36 & 0.06 & 0.78 & 0.10 & 0.22 & 0.35 & 0.15 & 0.03 \\
\hline $\mathrm{K}_{2} \mathrm{O}$ & 0.54 & 3.51 & 0.60 & 2.63 & 2.97 & 4.98 & 1.02 & 6.50 & 6.93 & 0.89 & 7.22 & 0.35 & 7.81 & 0.22 & 0.34 & 2.52 & - \\
\hline $\mathrm{TiO}_{2}$ & 0.22 & 0.27 & 0.05 & 0.01 & - & - & 0.01 & 0.08 & 0.01 & 0.05 & - & 0.12 & - & 0.0 & 0.13 & 0.07 & - \\
\hline $\mathrm{NiO}^{\circ}$ & - & 0.06 & - & 0.10 & - & - & 0.14 & - & - & 0.03 & 0.05 & - & - & 0.02 & - & - & 0.05 \\
\hline $\mathrm{Cr}_{2} \mathrm{O}_{3}$ & 0.01 & - & 0.01 & 0.08 & 0.11 & 0.27 & 0.08 & - & - & - & 0.01 & 0.04 & 0.01 & 0.04 & - & 0.02 & 0.07 \\
\hline $\mathrm{P}_{2} \mathrm{O}_{5}$ & 0.35 & 0.04 & 0.28 & - & 0.06 & 0.06 & - & - & - & - & 0.03 & - & - & - & 0.02 & 0.05 & 1.01 \\
\hline Total & 68.39 & 92.10 & 84.35 & 83.74 & 91.32 & 91.63 & 82.64 & 94.16 & 92.93 & 77.86 & 93.67 & 67.32 & 90.77 & 79.13 & 82.33 & 90.84 & 58.60 \\
\hline
\end{tabular}

Note: 1 = Sample 507C-3-1, 2-5 cm, orange material core of vesicle, 2 = Sample 507C-3-1, 2-5 cm, yellowish-green clay mineral in vesicle rim; 3 = Sample 508B-3-1, 12-15 cm, orange material in vesicle core; $4=$ Sample 508B-3-1, 12-15 cm, yellowish green clay mineral in a vesicle rim; $5=$ Sample 508B-3-1, 12-15 cm, brownish green clay mineral in vesicle; 6 = Sample 510-9-1, 44-46 cm, dark green clay mineral septum between two fresh olivine islets; 7 = Sample 510-9-1, 44-46 cm, pale green clay-mineral septum around the clay mineral analyzed in 6; $8=$ Sample 510-9-1, 44-46 cm, dark green clay mineral partly replacing olivine; $9=$ Sample $510-9-1,44-46 \mathrm{~cm}$, dark green clay-mineral replacing olivine rim; $10=$ Sample $510-9-1,44-46 \mathrm{~cm}$, pale green clay mineral replacing olivine core and associated with clay mineral analyzed in $9 ; 11=$ Sample $510-9-1,44-46 \mathrm{~cm}$, dark green clay mineral in a miarolitic void; 12 = Sample 510-9-1, 44-46 cm, pale brown clay mineral replacing olivine; 13 = Sample $510-9-3,31-36 \mathrm{~cm}$, dark green clay mineral partly replacing olivine; $14=$ Sample $510-9-3,31-36 \mathrm{~cm}$, pale brown clay mineral replacing olivine; 15 = Sample $510-9-3,31-36 \mathrm{~cm}$, pale greenish brown clay mineral replacing olivine; $16=$ Sample $510-9-3,31-36 \mathrm{~cm}$, dark brown clay mineral replacing olivine; $17=$ Sample $510-9-3,31-36 \mathrm{~cm}$, carbonate associated with dark green clay mineral and replacing olivine. Most analyses are representative of several made of optically similar materials, which gave practically identical results.

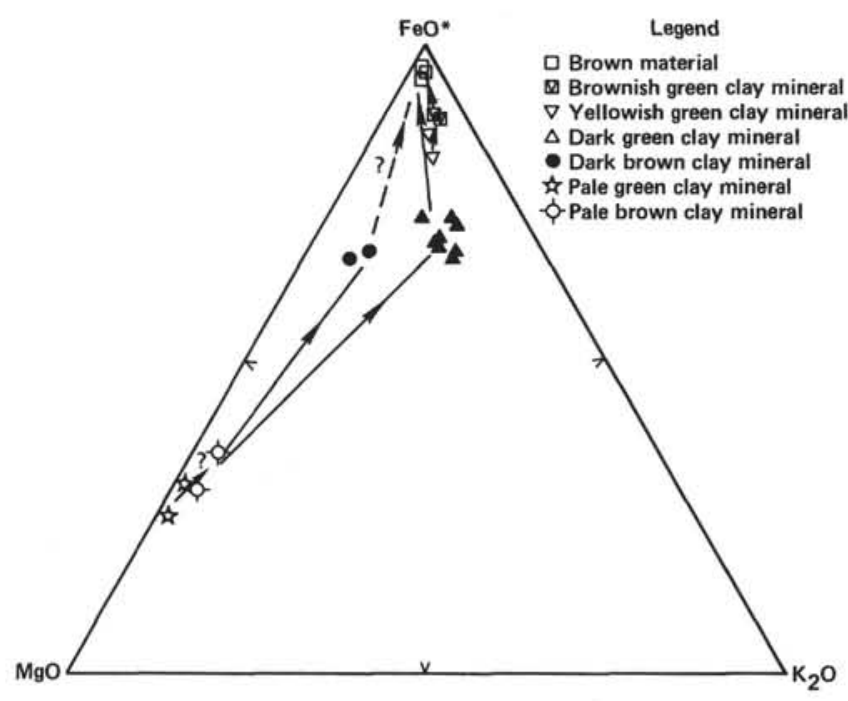

Figure 4. $\mathrm{FeO}^{*}-\mathrm{MgO}-\mathrm{K}_{2} \mathrm{O}$ diagram for secondary clay minerals in basalts. (Full $=$ Site 510 ; empty $=$ Sites $506,507,508$.)

The formation of these carbonate minerals may be explained by a longer period of interaction between the rocks and seawater.

The following secondary mineral sequence was observed on the ship (from the vein walls to the center): pyrite, various clays, and Fe-rich brown material. This indicates that conditions changed from reducing to oxidizing during alteration. But we did not observe this sequence in any of our samples.

\section{BULK-ROCK CHEMISTRY OF ALTERED BASALTS}

Most of the "fresh" samples we studied display altered halos. In this case, both fresh and altered rocks

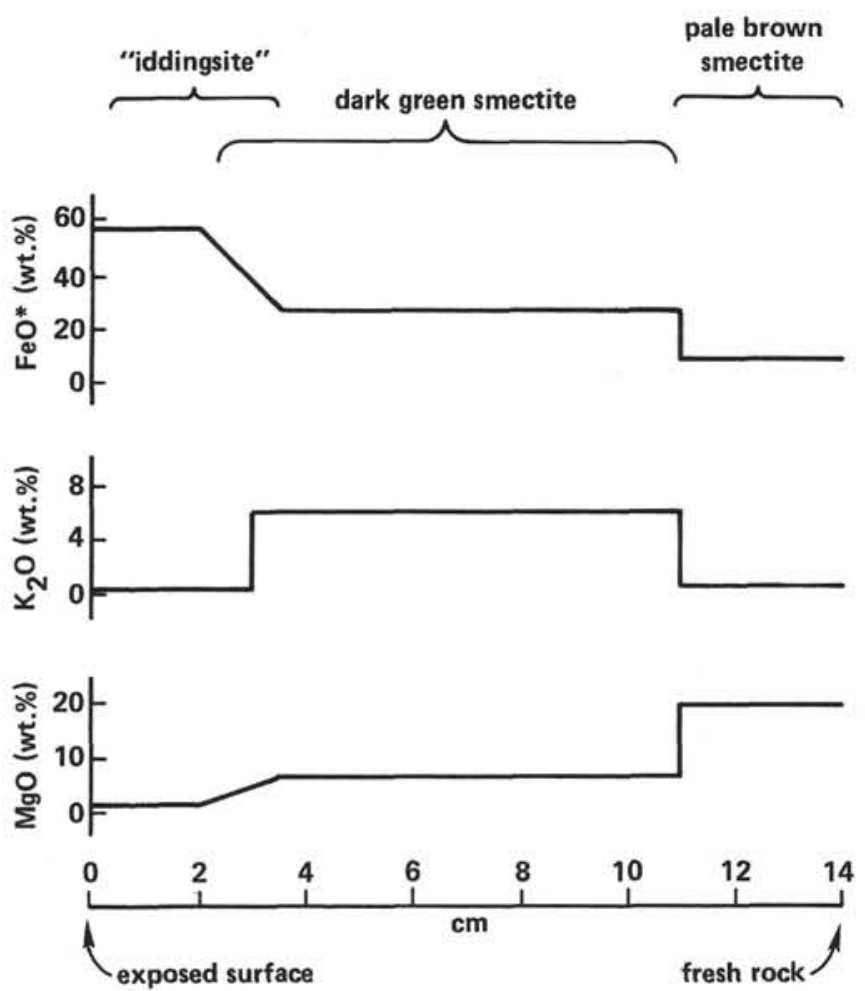

Figure 5. Variation of the $\mathrm{FeO}^{*}, \mathrm{~K}_{2} \mathrm{O}$, and $\mathrm{MgO}$ average contents. In clay minerals: distance to exposed surface.

have been separated and analyzed (Table 1). As the altered halos are zoned (see Fig. 3), the analyses represent the chemical composition of the entire altered area.

We have chosen to show chemical variations in the oxides in terms of their oxidation ratio $\left(\mathrm{O}_{\mathrm{x}}^{\circ}\right)$. This ratio is expressed by the atomic ratio of $\mathrm{Fe}^{3+} / \mathrm{Fe}^{\mathrm{T}}$. 
Representative points of pairs of fresh and altered rocks are connected by tie lines in Figures 6 and 7, which show the following:

1) A systematic oxidation of iron, with an $\mathrm{O}_{\mathrm{x}}^{\circ}$ gap ranging between 0.20 and 0.25 for "fresh" and altered rocks, respectively. The $\mathrm{Fe}$ content increases similarly (Table 3).

2) Alteration is generally accompanied by hydration. However, three samples (Samples 506G-2-1, 49-52 cm; $510-9-3,31-36 \mathrm{~cm}$; and $510-11-1,79-82 \mathrm{~cm}$ ) show the opposite trend-i.e., the fresh basalts are more hydrated than their altered rim. In such cases the "apparently fresh" basalt must have been hydrated before being oxidized. The explanation for this is unclear.

3) $\mathrm{K}_{2} \mathrm{O}$ content drastically increases with alteration. Fresh basalts contain from 0.04 to 0.24 wt. $\% \mathrm{~K}_{2} \mathrm{O}$, whereas altered rims contain 0.18 to 0.50 wt. $\% \mathrm{~K}_{2} \mathrm{O}$.

4) Most altered rims are slightly richer in Co than the fresh equivalents of the same pair. It is noticeable that the Co content in Sample 506G-2-1, 49-52 cm increased greatly during alteration $(193 \mathrm{ppm})$. The opposite was observed in Hole 396B of Leg 46 by Honnorez et al. (1978) for the brown rims where olivine was replaced by iron oxyhydroxides.

5) Fresh samples from Site 510 are very poor in $\mathrm{P}_{2} \mathrm{O}_{5}$. Thus, any transformation resulting from a fluid phase induces no perceptible effect, or it induces an increase in phosphorus during the first stage of alteration.

6) During alteration, content of all other elements decreases more or less. In fact, the more abundant an element is in the fresh basalt, the more it decreases with alteration.

7) Sample 506G-2-1, 49-52 $\mathrm{cm}$ behaves erratically: The fresh sample represents some kind of transition between basalts from Sites 507 and 508 and those from Site 510. Furthermore, during alteration, it often shows opposite trends with respect to other sites, with a clear gain of $\mathrm{Co}(90 \mathrm{ppm}) \mathrm{Ti}, \mathrm{Ni}$, and $\mathrm{V}$; a slighter gain of $\mathrm{Mg}$ ?, $\mathrm{Na}$, and $\mathrm{Al}$; no change in $\mathrm{Ca}$; and a loss of $\mathrm{K}$ and $\mathrm{H}_{2} \mathrm{O}$. This could be explained in terms of a primary compositional difference between "fresh" and altered samples.

8) Finally, an important gain in $\mathrm{Ni}$ occurs during alteration in Sample 507C-3-1, 2-5 cm.

In summary, the first alteration stage is hydration and oxidation. The apparent variations in oxide content during this alteration are: an increase in $\mathrm{H}_{2} \mathrm{O}, \mathrm{K}_{2} \mathrm{O}$, $\mathrm{FeO}^{*}$, and a slight decrease in $\mathrm{SiO}_{2}, \mathrm{Al}_{2} \mathrm{O}_{3}, \mathrm{MgO}, \mathrm{CaO}$, and $\mathrm{Na}_{2} \mathrm{O}$.

Various authors (Matthews, 1971; Hekinian, 1971; Thompson, 1973; Hart et al., 1974; Shido et al., 1974; Honnorez, in press) agree that the $\mathrm{Fe}^{3+} / \mathrm{Fe}^{2+}$ ratio and the content of $\mathrm{K}_{2} \mathrm{O}$ and $\mathrm{H}_{2} \mathrm{O}$ increase in oceanic basalts altered at low temperature. Most of the authors (Matthews, 1971; Thompson, 1973) also report that $\mathrm{Ca}, \mathrm{Mg}$, and $\mathrm{Si}$ decrease. Al content of altered rocks decreases according to Hart et al. (1974) and Thompson (1973), but remains unaffected according to most of the other authors (Hekinian, 1971; Hart, 1970; Thompson, 1973). The behavior of $\mathrm{Na}$ is very controversial.

In their study of alteration of pillow basalts in Hole 396B (IPOD, Leg 46), Honnorez et al. (1979) observed chemical variations. They calculated the values of these variations, assuming that $\mathrm{Al}_{2} \mathrm{O}_{3}$ is immobile during alteration. For them, an apparent gain of $\mathrm{FeO}^{*}, \mathrm{Al}_{2} \mathrm{O}_{3}$, $\mathrm{TiO}_{2}, \mathrm{CaO}$, and $\mathrm{Na}_{2} \mathrm{O}$ merely results from actual losses of $\mathrm{MgO}$ and $\mathrm{SiO}_{2}$. This normalization was possible because of the large number (77) of fresh basalts analyzed in Hole 396B and because of the homogeneity of the values.

It is not possible here to balance variations by an average $\mathrm{Al}_{2} \mathrm{O}_{3}$-content value for all sites because of the evolutive feature discussed in our study of fresh basalts.

Site by site, average $\mathrm{Al}_{2} \mathrm{O}_{3}$ content is $15.84 \mathrm{wt} . \%$ for Site $510,13.64$ wt. $\%$ for Site 506 , and 13.18 wt.\% for Sites 507 and 508. (The last two were grouped because of their compositional similarities.) Normalization of $\mathrm{Al}_{2} \mathrm{O}_{3}$ sheds light on the behavior of some elements. Increases of $\mathrm{H}_{2} \mathrm{O}, \mathrm{K}_{2} \mathrm{O}$, and $\mathrm{FeO}^{*}$ content persist, but the variations in content of $\mathrm{SiO}_{2}, \mathrm{MgO}, \mathrm{CaO}$, and $\mathrm{Na}_{2} \mathrm{O}$ become insignificant.

If one considers hydration to be the first alteration stage, changes in the concentrations of other elements can be estimated from normalized concentrations of cations on a wt. $\%$ water-free basis (Table 3). When we cancel the effects of hydration in this way, we find a significant gain in $\mathrm{Fe}$ and $\mathrm{K}$ and a slight loss of $\mathrm{Mg}$. The other element variations (i.e., $\mathrm{Si}, \mathrm{Ca}$, and $\mathrm{Na}$ ) show a very slight statistical decrease, which is not clearly significant. The small variations in $\mathrm{Al}$ content are more irregular, but the most important change is decreasing variations, mostly in basalts from Sites 508 and 510 . Potassium content can increase by a factor of five and shows the largest relative variations.

In conclusion, the first alteration stage of apparently fresh oceanic basalt is mainly characterized by oxidation and hydration, leading to a "dilution" of elements: an apparent decrease of $\mathrm{SiO}_{2}, \mathrm{MgO}$, and $\mathrm{CaO}$. The $\mathrm{K}_{2} \mathrm{O}$ rich fluid phase causes an increase in this element in the altered rock. The altered rims are also slightly more rich in $\mathrm{Fe}$.

A further alteration stage, observed in a "fresh" basalt which is already oxidized and hydrated, is mainly characterized by a loss of $\mathrm{SiO}_{2}$ and $\mathrm{MgO}$ (Honnorez et al., 1979). In this case, the $\mathrm{K}_{2} \mathrm{O}$ behavior during alteration becomes erratic, but potassium content tends to increase.

\section{GENERAL CONCLUSION}

Young crust from the Galapagos Spreading Center (Sites 506, 507, and 508) is represented by a fine- to medium-grained basalt with some plagioclase and clinopyroxene phenocrysts, abundant titanomagnetite, but no olivine. Site 510 basement, which is older, is represented by a fine- to medium-grained, plagioclase, $\mathrm{Mg}$ rich olivine phyric basalt. Titanomagnetite is less abundant. Phenocryst assemblages, $\mathrm{Fe}-\mathrm{Ti}$ oxide abundance, and bulk-rock chemistry indicate that Site 510 basalts are more primitive than are those from Sites 506, 507, and 508. Comparison with a previous study (Fodor et al., 1980) of basalts from Leg 54 (Sites 424 and 425) confirms that the composition of Site 510 basalts is very close to that of the tholeiitic parental magma. Fractionation increases from Site 510, 425, 506, 507, and 508 

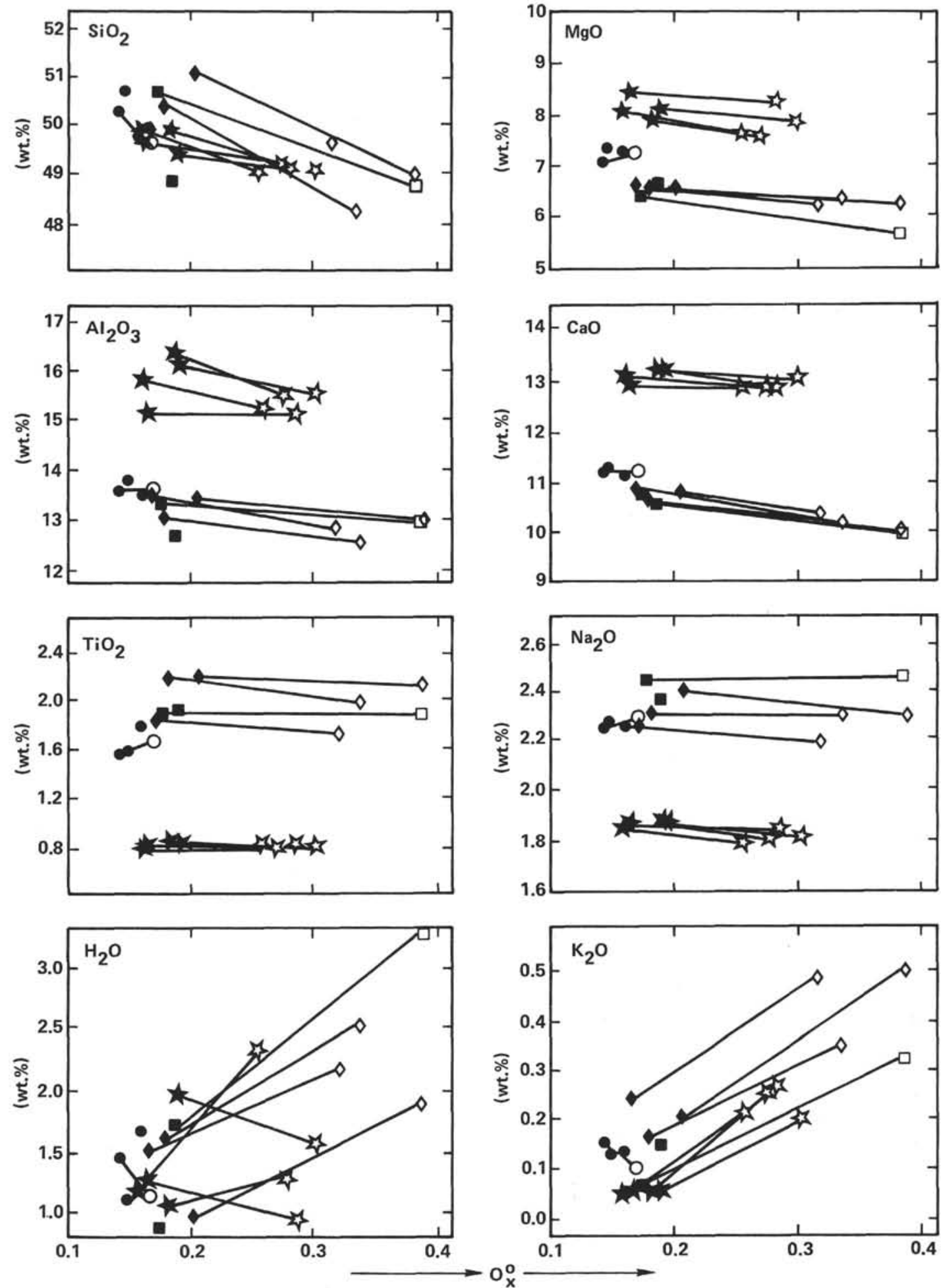

Legend $O=$ Site $506 \square=$ Site $507 \Delta=$ Site $508 \quad$ = Site $510 \Delta=$ Site $424 \nabla=$ Site 425

Black symbol $=$ fresh rock; empty symbol $=$ altered rim

Figure 6. Bulk-chemical variations with alteration: weight oxides vs. $\mathrm{O}_{\mathrm{x}}^{\circ}$.

to Site 424 , and results in ferrobasalts in the last three sites. The drastic decrease of $\mathrm{Al}_{2} \mathrm{O}_{3}, \mathrm{MgO}, \mathrm{CaO}, \mathrm{Cr}$, and $\mathrm{Cu}$ and the important increase of $\mathrm{FeO}^{*}, \mathrm{TiO}_{2}, \mathrm{~V}$, and $\mathrm{Na}_{2} \mathrm{O}, \mathrm{Sr}, \mathrm{Ba}$ indicate fractionation of olivine and spinel plus plagioclase from the primitive tholeiite. Notice that the more evolved basalts are youngest.
Alteration of these basalts appears as dark rims, whose thickness increases with age of basalts from Site 506 to Sites 508 and 510 . The most common and abundant minerals in the altered rims are clay minerals (probably smectites), occurring in vesicles, miarolitic voids, and, eventually, as olivine replacement (Site 510). 

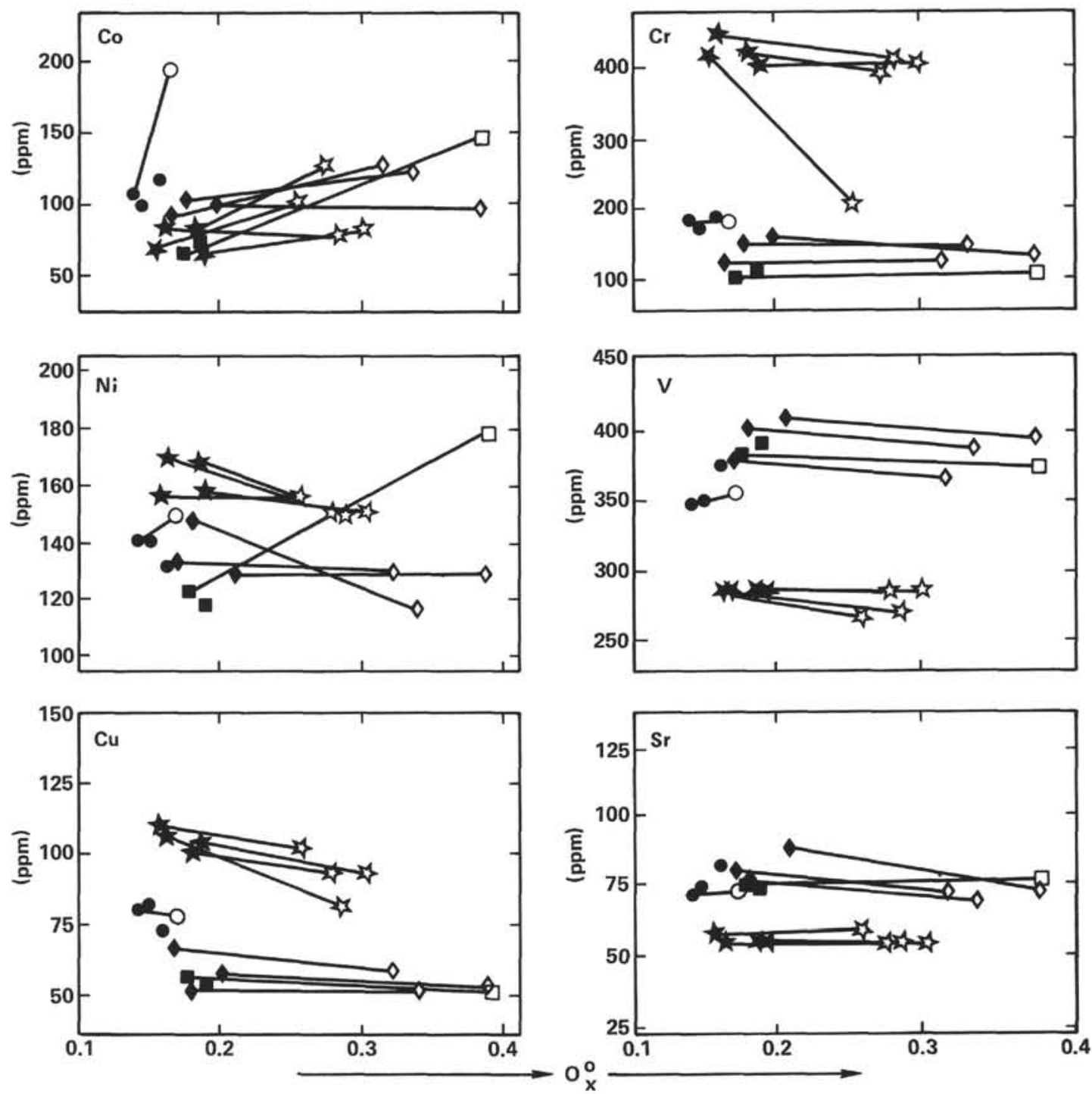

Legend $O=$ Site $506 \square=$ Site $507 \quad Q=$ Site $508 \quad$ = Site $510 \Delta=$ Site $424 \nabla=$ Site 425 Black symbols $=$ fresh rock; empty symbols $=$ altered rim

Figure 7. Bulk-chemical variations with alteration: $p p m$ vs. $\mathrm{O}_{\mathrm{x}}^{\circ}$

The altered rims show concentric zonation, marked by different types of minerals, indicating that the alteration of Galapagos Spreading Center basalts is characterized by (at least) three stages:

1) A reducing stage (pyrite formation), also found by Honnorez et al. (1978) in basalts from Leg 46, Hole 396B.

2) A nonoxidizing or slightly oxidizing stage (with formation of various clay minerals, particularly those that are K-rich), together with hydration.

3) An oxidizing stage ("iddingsite" and brownish green clay mineral formation), during which an oxidation front moves toward the innermost parts of the altered rim.

One can distinguish young basalts (Sites 506, 507, and 508) from older ones (Site 510) in terms of alteration: the oldest basalts contain very K-rich dark olive clay minerals, very K-poor pale brown clay minerals, and carbonates, whereas young basalts contain moderately rich-K, yellowish green clay mineral, and little or no carbonates (Site 508). This can be explained by tooshort interaction between basalt and seawater. Variation in the bulk-rock chemical composition indicates that the first alteration stage of apparently fresh oceanic basalts is mainly characterized by oxidation and hydration, which leads to an apparent "dilution" of elements. After normalization on a water-free basis, we find a significant gain in $\mathrm{Fe}$ and $\mathrm{K}$ and a slight loss of $\mathrm{Mg}$ in the altered rocks.

\section{ACKNOWLEDGMENT}

We want to thank M. Bohn for his help during the electron microprobe analyses. We also thank D. Shaw and T. Juteau for reviewing this paper, and J. Honnorez for his critical comments. 


\section{REFERENCES}

Anderson, R. N., Clague, D. A., Klitgord, K. D., Marshall, M., and Nishimori, R. K., 1975. Magnetic and petrologic variations along the Galapagos Spreading Center and their relation to the Galapagos melting anomaly. Geol. Soc. Am. Bull., 86:683-694.

Bohlke, J. F. P., 1978. Alteration of deep-sea basalts from Site 396B [M.S. thesis]. University of Miami, Miami.

Clague, D. A., and Bunch, T. E., 1976. Formation of ferrobasalt at East Pacific Midocean Spreading Centers. J. Geophys. Res., 81(23):4247-4256.

Deer, W. A., Howie, R. A., and Zussman, J., 1963. Rock Forming Minerals: London (Longmans).

Fodor, R. V., Berkley, J. L., Keil, K., Hustler, J. W., Ma, M.-S., and Schmitt, R. A., 1980. Petrology of basalt drilled from the Galapagos Spreading Center, DSDP Leg 54. In Rosendahl, B. R., Hekinian, R., et al., Init. Repts. DSDP, 54: Washington (U.S. Govt. Printing Office), 737-750.

Frey, F. A., Bryan, W. B., and Thompson, G., 1974. Atlantic Ocean floor: Geochemistry and petrology of basalts from Legs 2 and 3 of the Deep Sea Drilling Project. J. Geophys., 79:5507-5527.

Hart, R. A., 1970. Chemical exchange between seawater and deep ocean basalts. Earth Planet. Sci. Lett., 9:269-279.

1973. A model for chemical exchange in the basalt-seawater systems of oceanic layer II. Can. J. Earth Sci., 10:799-816.

Hart, S. R., Erlank, A. J., and Kable, E. J. D., 1974. Seafloor basalt alteration: Some chemical and $\mathrm{Sr}$ isotopic effects. Contrib. Mineral. Petrol., 44:219-230.

Hekinian, R., 1971. Chemical and mineralogical differences between abyssal hill basalts and ridge tholeites in the Eastern Pacific Ocean. Mar. Geol., 11:77-91.

Honnorez, J., in press. The oceanic lithosphere. In Emiliani, C. (Ed.), The Sea (Vol. 7): New York (Wiley).

Honnorez, J., Bohlke, J. K., and Honnorez-Guerstein, B. M., 1978. Petrographical and geochemical study of the low-temperature sub- marine alteration of basalt from Hole 396B, Leg 46. In Dmitriev, L., Heirtzler, J., et al., Init. Repts. DSDP, 46: Washington (U.S. Govt. Printing Office), 299-329.

Matthews, D. H., 1971. Altered basalts from Swallow Bank, an abyssal hill in the N.E. Atlantic and from a nearby seamount. Philos. Trans. R. Soc. London, Ser. A., 268:551-571.

Melson, W. G., Vallier, T. L., Wright, T. L., Byerly, G., and Nelen, J., 1976. Chemical diversity of abyssal volcanic glass erupted along Pacific, Atlantic and Indian Ocean seafloor spreading centers. Am. Geophys. Union Monograph, 19:351-367.

Mevel, C., 1980. Mineralogy and chemistry of secondary phases in low temperature altered basalts from DSDP Legs 51, 52, 53. In Donnelly, T., Francheteau, J., Bryan, W., Robinson, P., Flower, M., Salisbury, M., et al., Init. Repts. DSDP, 51, 52, 53, Pt. 2: Washington (U.S. Govt. Printing Office), 1299-1318.

Miyashiro, A., 1974. Volcanic rock series in island arcs and active continental margins. Am. J. Sci., 274:321-355.

Schilling, J. G., Anderson, R. N., and Vogt, P., 1976. Rare earth, $\mathrm{Fe}$ and $\mathrm{Ti}$ variations along the Galapagos Spreading Center, and their relationship to the Galapagos mantle plume. Nature, 261: 108-113.

Shido, F., Miyashiro, A., and Ewing, M., 1974. Compositional variation in pillow lavas from the Mid-Atlantic Ridge. Mar. Geol., 16:177-190.

Thompson, G., 1973. A geochemical study of the low-temperature interaction of seawater and oceanic igneous rocks. Eos, 54: 1015-1019.

Thompson, G., Bryan, W. B., Frey, F. A., Dickey, T. S., and Suen, C. J., 1976. Petrology and geochemistry of basalt from DSDP Leg 34, Nazca Plate. In Yeats, R. S., Hart, S. R., et al., Init. Repts. DSDP, 34: Washington (U.S. Govt. Printing Office), 215-226.

Wilshire, H. G., 1958. Alteration of olivine and orthopyroxene in basic lavas and shallow intrusions. Am. Mineral., 43:120-145. 

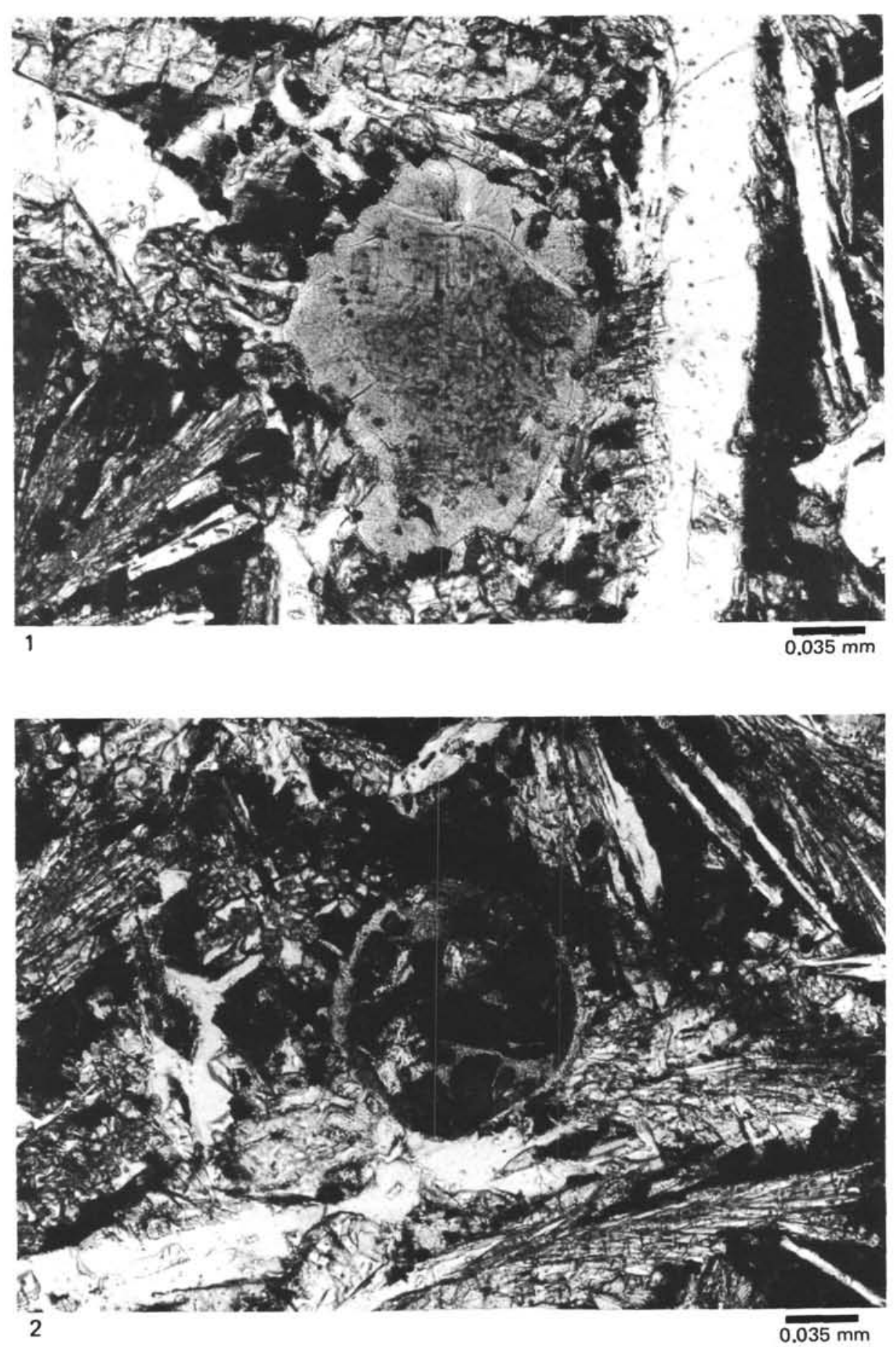

Plate 1. Portions of zonational pattern in altered rim (plane polarized light); Sample 508B-3-1, 12-15 cm. 1. Vesicle with yellowish green clay mineral. 2. Vesicle filled with brown material, yellowish green clay mineral. (Scale bar $=0.035 \mathrm{~mm}$.) 

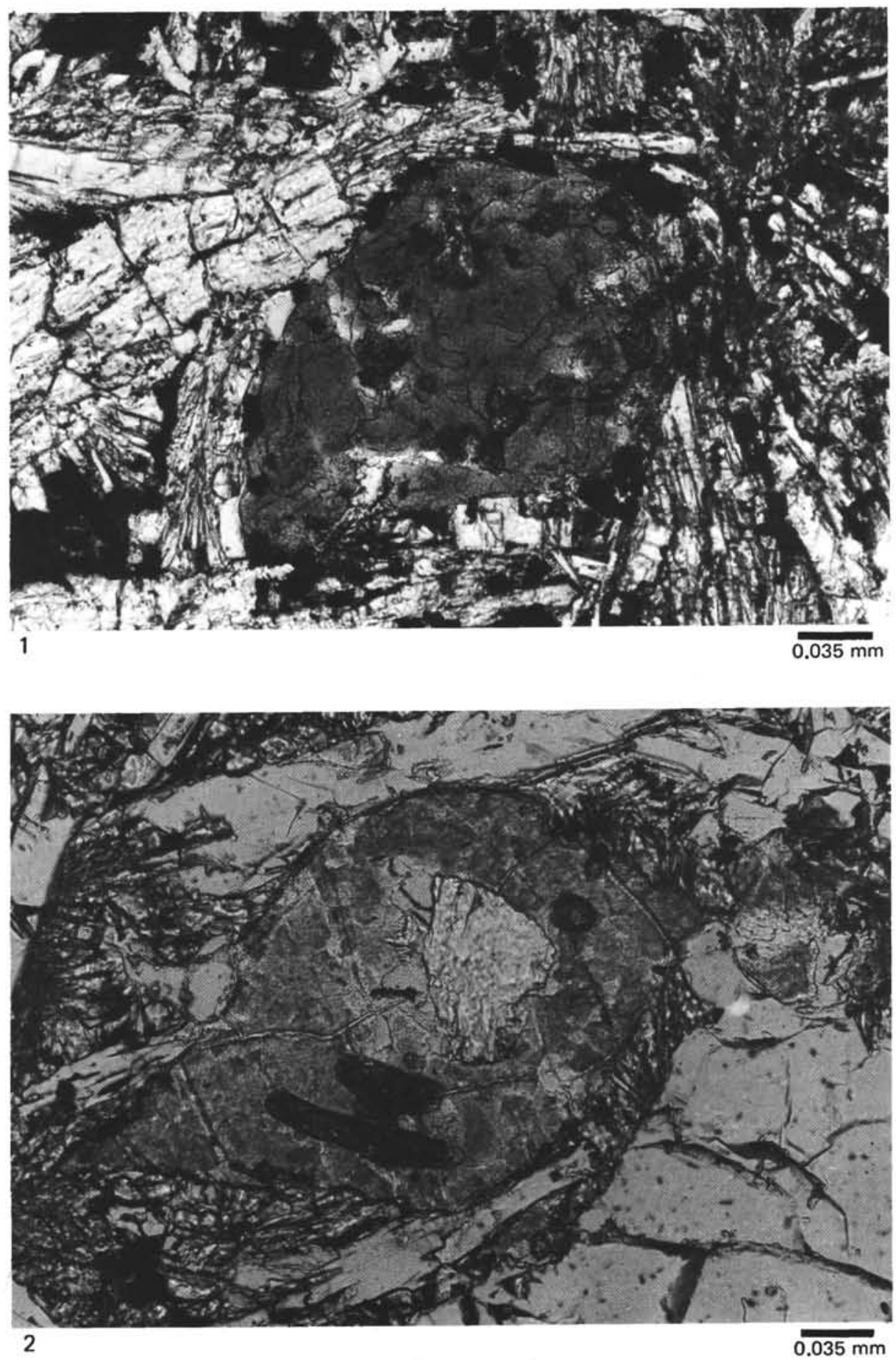

Plate 2. Portions of zonational pattern in altered rim (plane polarized light); Sample 510-9-3,31-36 cm. 1. Olivine microphenocryst replaced by dark green clay mineral. 2. Olivine microphenocryst completely replaced by dark green clay mineral "iddingsite" (brown material) close to a thin "iddingsite" vein. (The differences in color of the materials cannot be distinguished here.) (Scale bar $=0.035 \mathrm{~mm}$.) 

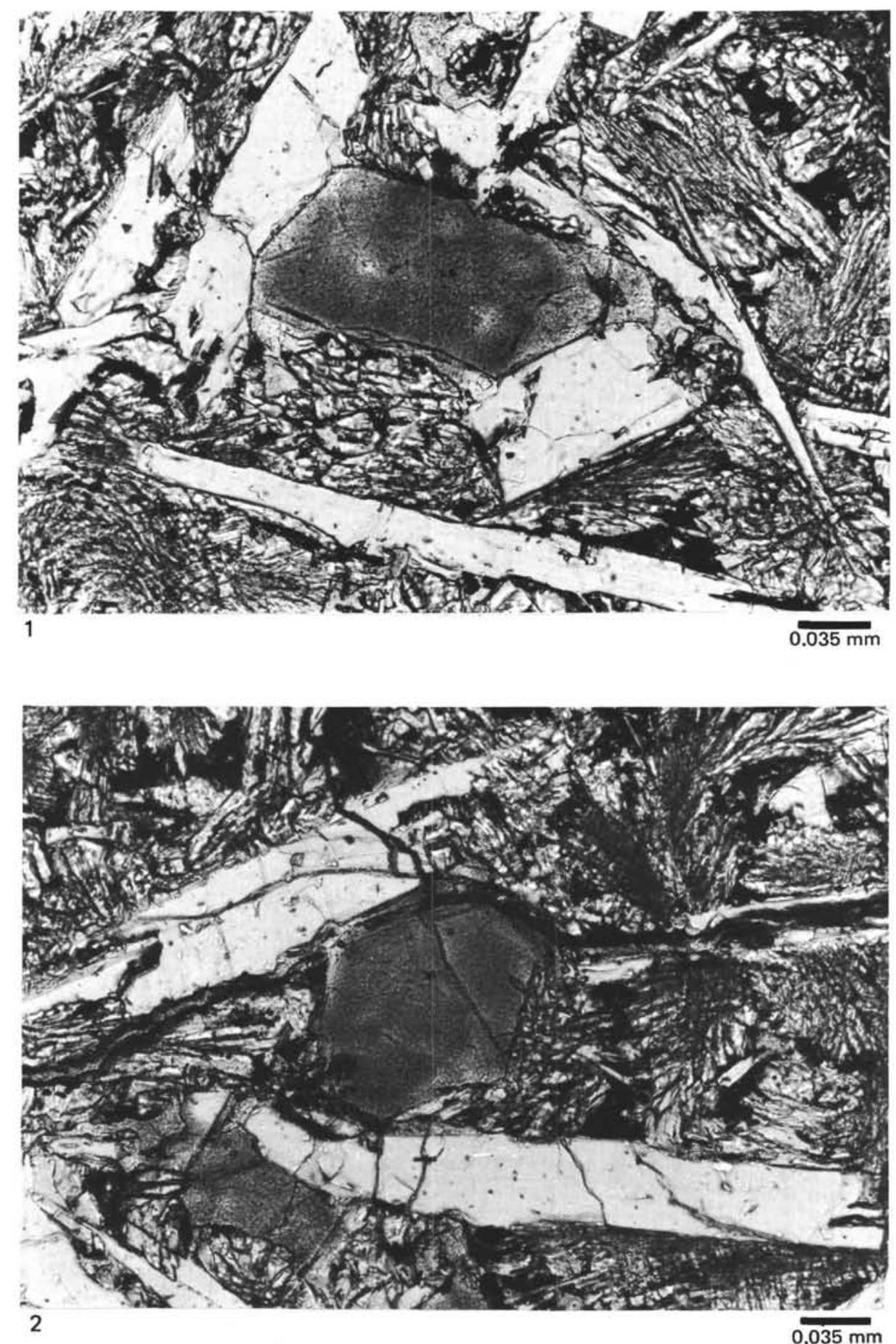

Plate 3. Portions of zonational patterns in two altered rims (plane polarized light). 1. Sample 508B-3-1, 12-15 cm; vesicle filled with brown material. 2. Sample $510-9-3,31-36 \mathrm{~cm}$; olivine phenocryst completely replaced by pale brown clay mineral carbonate. (Scale bar $=0.035 \mathrm{~mm}$.) 